J. Math. Biol. (2012) 64:1043-1086

DOI 10.1007/s00285-011-0439-z

\title{
COMPETITION IN THE PRESENCE OF A VIRUS IN AN AQUATIC SYSTEM: AN SIS MODEL IN THE CHEMOSTAT
}

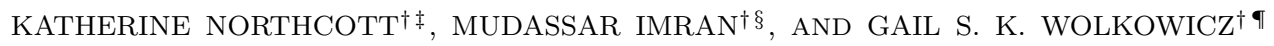

\begin{abstract}
Recent research indicates that viruses are much more prevalent in aquatic environments than previously imagined. We derive a model of competition between two populations of bacteria for a single limiting nutrient in a chemostat where a virus is present. It is assumed that the virus can only infect one of the populations, the population that would be a more efficient consumer of the resource in a virus free environment, in order to determine whether introduction of a virus can result in coexistence of the competing populations. We also analyze the subsystem that results when the resistant competitor is absent. The model takes the form of an SIS epidemic model. Criteria for the global stability of the disease free and endemic steady states are obtained for both the subsystem as well as for the full competition model. However, for certain parameter ranges, bi-stability, and/or multiple periodic orbits is possible and both disease induced oscillations and competition induced oscillations are possible. It is proved that persistence of the vulnerable and resistant populations can occur, but only when the disease is endemic in the population. It is also shown that it is possible to have multiple attracting endemic steady states, oscillatory behavior involving Hopf, saddle-node, and homoclinic bifurcations, and a hysteresis effect. An explicit expression for the basic reproduction number for the epidemic is given in terms of biologically meaningful parameters. Mathematical tools that are used include Lyapunov functions, persistence theory, and bifurcation analysis.
\end{abstract}

Key words. SIS epidemic model, lytic and lysogenic virus, Hopf, homoclinic, and saddle-node bifurcations, bi-stability and hysteresis effect, multiple limit cycles, biodiversity

AMS subject classifications. 34C60, 34C23, 92D25, 93D30, 93D20, 34C37, 34C55, 34C15

1. Introduction. It is known that bacteria are present in abundance in marine and lake environments, but it was only recently discovered that viruses are present in even greater abundance than bacteria [6]. Indeed, current research seems to indicate that viruses have significant impact on bacterial populations in aquatic environments and may be responsible for controlling phytoplankton blooms in the ocean and they may play a role in regulating biodiversity [19]. These special viruses that attack bacteria are called bacteriophage or phage. In a letter to Nature, Bergh et al. [5] state that "...virus infection may be an important factor in the ecological control of planktonic micro-organisms..." and indicate that studying the role of viruses in aquatic environments should not be neglected. They suggest that by enhancing bacterial diversity, a phage can act as a "controller". Phage usually have a specificity with regard to their prey, and so they often attack the otherwise "stronger" competitor, thus reducing the effect of phytoplankton and phytobacterial blooms in the ocean by severely inhibiting the competitive capacity of the blooming microorganism, allowing "lesser" competitors to step in ([6], [7], [31]). Other studies ([11], [19], [23]) also provide support that viruses and bacteriophage play a significant role in aquatic bacterial ecology.

There are two typical methods of viral reproduction, the lytic cycle considered virulent and the lysogenic cycle considered temperate [21]. In [32], the proliferation of temperate viruses in Lake Superior was studied. Campbell [9] and Lwoff [21] also report on the widespread nature and significance of lysogenic phage. The lytic cy-

${ }^{\dagger}$ Department of Mathematics and Statistics, McMaster University, Hamilton, Ontario, Canada L8S 4K1

$\ddagger$ e-mail: katherine.northcott@gmail.com

$\S$ e-mail: mimran@math.mcmaster.ca

ฯ corresponding author, e-mail: wolkowic@mcmaster.ca, telephone: (905) 525-9140 x24808, fax: (905) 522-0935, research partially supported by NSERC 
cle involves four stages: penetration of the host cell; biosynthesis using the host cell to manufacture large amounts of viral components; biosynthesis i.e., assembling the viral components into complete viruses; and lysis or bursting of the infected cell releasing new viruses that infect other cells. However, some lytic viruses escape the host cell without bursting the cell membrane, by budding off taking a portion of the cell membrane from the host cell. In the lysogenic cycle, the bacteriophage does not immediately destroy the hosts DNA and take control of the cell. Similar to lytic reproduction, lysogenic reproduction also begins when the phage inserts its DNA or RNA into the host cell through its surface. Once inside the cell, it becomes a part of the genome of the host cell and is called a prophage. The phages genetic information is copied and distributed to the daughter cells of the host cell, which continues dividing naturally. This allows the prophage to be repeatedly copied and passed on without destroying the host cell it depends on for metabolic and reproduction purposes. In order for actual active phages to be produced, the segment of genome from the original phage exits the genome of the host cell and becomes independent. When this occurs, it begins the lytic cycle, destroying the cell, but producing new and functional phages. After becoming lysogenic, some bacteria lose their lysogenic power, becoming newly susceptible. Often bacteriophage use a mixed strategy (see [10]). Such bacteria/viruses are most relevant here.

The basic chemostat model predicts that coexistence of two or more microbial populations competing for a single non-reproducing nutrient is not possible. (See ([1], [8], [14], [15], [20], [38]). This prompts the question: Can the incorporation of a virus induce the stable coexistence of bacterial competitors in a chemostat-like environment?

We consider a model of exploitative competition in a chemostat containing a virus. We assume that only one of the competing populations is susceptible to infection by the virus, the population that would be the superior competitor in the absence of the virus. We model the dynamics of the infection using an SIS model, i.e. bacteria become infected with the virus by close contact with other infected bacteria or bacteria that burst. We assume that not all the infected cells lyse, but rather some are able to clear the virus and return to the normal susceptible state. Thus there can be both lytic and lysogenic cycles. Since we assume an infectious contact rate between the susceptible and infected population, as in most epidemic models, we assume that it is not necessary to model the virus explicitly. The model of the vulnerable population takes the form of a standard SIS epidemic model and so the results of our analysis would apply to any SIS disease in a chemostat. Our model reduces to the SI model when one of the parameters, the parameter modeling recovery, is set to zero.

Other studies that consider the effect of a virus on competing species in a basic chemostat include Mestivier et al. [22] and Weitz et al. [35]. Unlike our model, they model the virus explicitly and do not allow recovery, i.e., they only consider an SI model. In both studies a linear analysis is given. In [22] simulations show that coexistence between two bacterial populations can be induced by the addition of a virulent virus. In [35] it was shown that for a reasonable choice of parameters, the system possesses a coexistence steady state. They also demonstrated coexistence using Monte Carlo simulations of populations evolving in a chemostat.

For other related mathematical models of phage-bacteria interaction see ([4], [24]), [27]). Beretta [3] discussed both deterministic and stochastic models for phage bacteria infection in an open marine environment. Mestivier et al. [22] investigate viruscoerced coexistence and diversity in marine bacteria and Shabir et al. [26] studied 
the coexistence in wastewater treatment in an upflow anaerobic sludge bed reactor treating sulfate-rich wastewater.

We begin by introducing the model in $\S 2$ and discuss its various subsystems. We then summarize our results in $\S 3$. After considering the number and stability (local and global) of the various equilibria analytically, we explore the bifurcations of the equilibria using numerical continuation software XPPAUT [13] to demonstrate Hopf, transcritical, and homoclinic bifurcations, saddle-node bifurcations of equilibria and of periodic orbits resulting in more than one limit cycle, and a hysteresis effect. We show that for certain ranges of the parameters initial condition dependent outcomes (bi-stability) is possible, e.g., it is possible to have multiple stable endemic steady states or a stable endemic steady state and a stable periodic attractor. Since it is not always the case that there is a globally asymptotically stable equilibrium, we use persistence theory to obtain criteria that predicts which populations survive, independent of the initial conditions. In particular, we obtain sufficient conditions for the persistence of both the susceptible and the resistant populations, thus showing that introducing disease can enhance diversity, since in the absence of disease at most one competitor population can survive. We demonstrate that this persistence of both the susceptible and the resistant populations (provided the disease is endemic) can either be in the form of convergence to an asymptotically stable steady state or in the form of sustained oscillatory behavior. We demonstrate that there can be both disease induced oscillations (i.e., oscillatory behavior is possible even when the resistant population is absent) and competition induced oscillations (i.e., invasion by the resistant population can result in sustained oscillations even if there are no oscillations in its absence). We summarize our results and discuss certain implications in the discussion in $\S 4$. A subsystem of our model was studied as a model of conjugationally transmitted plasmids in bacterial populations (see Imran and Smith [17] and Stewart and Levine [30]). Implications of our analysis in this context are described. For clarity of the presentation, proofs are relegated to the appendices $\S \mathrm{A}, \mathrm{B}$, and $\mathrm{C}$.

2. The Model. We considered a model that involves two species that compete exploitatively for a single non-reproducing growth-limiting nutrient, in a well-stirred chemostat in the presence of a virus. In the growth chamber the concentration at time $t$ is denoted by $S(t)$. One species $x$, is susceptible to attack by the virus. This species is divided into two subpopulations, susceptibles with concentration at time $t$ denoted by $x_{s}(t)$ and infectives (bacteriophage) with concentration denoted by $x_{I}(t)$. The second species with concentration denoted by $y(t)$, is not susceptible to attack by the virus. Since the virus requires a host to replicate, we do not model the virus explicitly, but rather we assume that the virus is spread when infected bacteria lyse close to susceptible bacteria. The disease dynamics are therefore modelled in the form of an SIS epidemic model.

We analyze this system with particular interest in determining under what conditions coexistence of all three populations $x_{s}, x_{I}$, and $y$ is possible. More specifically, we consider the following model:

$$
\begin{aligned}
S^{\prime}(t)= & \left(S^{0}-S(t)\right) D-\frac{\alpha_{s} x_{s}(t) S(t)}{\eta_{s}}-\frac{\alpha_{I} x_{I}(t) S(t)}{\eta_{I}}-\frac{\alpha_{y} y(t) S(t)}{\eta_{y}} \\
x_{s}^{\prime}(t)= & x_{s}(t)\left(-D_{s}+\alpha_{s} S(t)\right)-\delta x_{s}(t) x_{I}(t)+\gamma x_{I}(t) \\
x_{I}^{\prime}(t)= & x_{I}(t)\left(-D_{I}+\alpha_{I} S(t)\right)+\delta x_{s}(t) x_{I}(t)-\gamma x_{I}(t) \\
y^{\prime}(t)= & y(t)\left(-D_{y}+\alpha_{y} S(t)\right) \\
& \text { with } \quad S(0) \geq 0, x_{s}(0) \geq 0, x_{I}(0) \geq 0, \quad \text { and } \quad y(0) \geq 0 .
\end{aligned}
$$


Here, $S^{0}$ denotes the concentration of the growth-limiting nutrient in the nutrient reservoir and $D$ the rate of inflow from the nutrient reservoir to the growth chamber as well as the rate of outflow from the growth chamber (hence the volume in this vessel remains constant); $D_{s}, D_{I}$, and $D_{y}$ denote the sum of the species-specific death rate and the rate of outflow of $x_{s}(t), x_{I}(t)$, and $y(t)$ respectively; and for $x_{s}(t)$, $x_{I}(t)$, and $y(t)$ respectively, $\alpha_{s}, \alpha_{I}$, and $\alpha_{y}$ denote the growth coefficients; $\eta_{s}, \eta_{I}$, and $\eta_{y}$ the growth yield constants (i.e. representing the conversion of nutrient to biomass); $\delta$ the rate of infection of susceptibles assumed related to susceptibles being in close proximity to infected bacteria that burst releasing phage; and $\gamma$ the rate of elimination or neutralization of virus (probably very small). We keep the model as simple as possible in order to focus on the effect of disease on the competitive outcome. Since without disease, coexistence of competing populations is not possible in the basic chemostat model for a general class of response functions (monotone and unimodal) including Holling type I, II, III and IV (see [38]), and we wish to investigate whether introduction of a disease can result in coexistence and even persistence, we assume linear response functions (mass action interactions), since coexistence would be least likely in his case.

It is natural to assume infection by the virus is detrimental to the $x_{I}$ population (as discussed in [9] and [35]), and so this determines the relative values of the parameters. It is assumed throughout that $x_{I}$ has a higher death rate (e.g., due to lysis) than $x_{s}$, so that $D_{I}>D_{s}$ and that its growth coefficient is no better than that of $x_{s}$, so that $\alpha_{s} \geq \alpha_{I}$. Hence, $\frac{D_{s}}{\alpha_{s}}<\frac{D_{I}}{\alpha_{I}}$. As for $y$, we will show that for all species to coexist, it is necessary to assume that $y$ is a weaker competitor for the nutrient than $x_{s}$ i.e. $\frac{D_{y}}{\alpha_{y}}>\frac{D_{s}}{\alpha_{s}}$.

If $\gamma=0$, system (2.1) becomes an SI model in the chemostat. This model was analyzed with a different interpretation in [12] and [37]. It was interpreted as a predator-prey model where the predator $x_{I}$ not only predated on $x_{s}$, but also competed with $x_{s}$ for the nutrient $S$.

2.1. Subsystems. There are two subsystems of (2.1) that are of interest. If $x_{I}(0)=0$, then $x_{I}(t) \equiv 0$ and we have the following virus-free subsystem:

$$
\begin{aligned}
S^{\prime}(t)= & \left(S^{0}-S(t)\right) D-\frac{\alpha_{s} x_{s}(t) S(t)}{\eta_{s}}-\frac{\alpha_{y} y(t) S(t)}{\eta_{y}} \\
x_{s}^{\prime}(t)= & x_{s}(t)\left(-D_{s}+\alpha_{s} S(t)\right) \\
y^{\prime}(t)= & y(t)\left(-D_{y}+\alpha_{y} S(t)\right) \\
& \text { with } \quad S(0) \geq 0, \quad x_{s}(0) \geq 0, \quad \text { and } \quad y(0) \geq 0 .
\end{aligned}
$$

This model is a special case of the model analyzed in [38], where it was proved that, at most, one species can survive. More specifically, if $\frac{D_{s}}{\alpha_{s}} \geq S^{0}$ then $x_{s}(t) \rightarrow 0$ as $t \rightarrow \infty$. If $\frac{D_{y}}{\alpha_{y}} \geq S^{0}$ then $y(t) \rightarrow 0$ as $t \rightarrow \infty$. On the other hand, if $\frac{D_{s}}{\alpha_{s}}<\min \left(S^{0}, \frac{D_{y}}{\alpha_{y}}\right)$ then $x_{s}(t)$ is the sole survivor, whereas if $\frac{D_{y}}{\alpha_{y}}<\min \left(S^{0}, \frac{D_{s}}{\alpha_{s}}\right)$ then $y(t)$ is the sole survivor. Coexistence is not possible. If $y(0)=0$, then $y(t) \equiv 0$ and we have the following subsystem:

$$
\begin{aligned}
& S^{\prime}(t)=\left(S^{0}-S(t)\right) D-\frac{\alpha_{s} x_{s}(t) S(t)}{\eta_{s}}-\frac{\alpha_{I} x_{I}(t) S(t)}{\eta_{I}} \\
& x_{s}^{\prime}(t)=x_{s}(t)\left(-D_{s}+\alpha_{s} S(t)\right)-\delta x_{s}(t) x_{I}(t)+\gamma x_{I}(t) \\
& x_{I}^{\prime}(t)=x_{I}(t)\left(-D_{I}+\alpha_{I} S(t)\right)+\delta x_{s}(t) x_{I}(t)-\gamma x_{I}(t)
\end{aligned}
$$


with $\quad S(0) \geq 0, x_{s}(0) \geq 0, \quad$ and $\quad x_{I}(0) \geq 0$.

Subsystem (2.2) was introduced by Stewart and Levin [30] (using different notation) to describe the dynamics of conjugationally transmitted plasmids in bacterial populations. Population $x_{I}$ represented the plasmid bearing bacteria and $x_{s}$ the plasmid free bacteria. In the context of our model, they considered the existence and local stability of equilibria in the special case that $D=D_{s}=D_{I}, \eta_{I}<\eta_{s}$, and $\alpha_{s} \approx \alpha_{I}$. Imran and Smith [17] studied the global stability of equilibria of this subsystem assuming $D=D_{s}=D_{I}$ and $\eta_{I}=\eta_{s}$.

We include the local and global analysis of this subsystem in this paper, since much of the analysis of (2.1) is based on understanding this particular subsystem. Relaxing the assumption that $D=D_{s}=D_{I}$ and the restriction on the relative values of the yield constants requires more delicate analysis than previously done in [17] and [30], and results in differences in the number of possible equilibria and the types of bifurcations that they can undergo.

If $x_{I}(0)>0$, a subsystem of $(2.1)$ with $x_{s}(t) \equiv 0$ is not possible, since if $x_{s}(0)=0$ but $x_{I}(0)>0$, then $x_{s}^{\prime}(0)=\gamma x_{I}(t)>0$. As well, no subsystem is possible without $S$. Hence, these are the only two subsystems that are of interest.

\section{Results.}

3.1. Well-posedness and Competition Independent Extinction. First we note that the model is well-posed. The proof is standard and hence omitted.

LEMma 3.1. All solutions of system (2.1) and subsystem (2.2) are bounded and remain non-negative for all $t>0$. Moreover, the set

$$
\mathcal{S} \equiv\left\{\left(S, x_{s}, x_{I}, y\right): S, x_{s}, x_{I}, y \geq 0 ; 0 \leq S+\frac{A}{\alpha}\left(x_{s}+x_{I}+y\right) \leq S^{0}\right\}
$$

is positively invariant and is a global attractor for (2.1), where $A=\min \left\{\frac{\alpha_{s}}{\eta_{s}}, \frac{\alpha_{I}}{\eta_{I}}, \frac{\alpha_{y}}{\eta_{y}}\right\}$ and $\alpha=\max \left\{\alpha_{s}, \alpha_{y}\right\}$, and

$$
\mathcal{S}_{(2.2)} \equiv\left\{\left(S, x_{s}, x_{I}\right): S, x_{s}, x_{I} \geq 0 ; 0 \leq S+\frac{A_{(2.2)}}{\alpha_{(2.2)}}\left(x_{s}+x_{I}\right) \leq S^{0}\right\},
$$

is positively invariant and is a global attractor for (2.2), where $A_{(2.2)}=\min \left\{\frac{\alpha_{s}}{\eta_{s}}, \frac{\alpha_{I}}{\eta_{I}}\right\}$ and $\alpha_{(2.2)}=\alpha_{s}$.

This next result shows that the concentration in the reservoir must be sufficiently high in order for survival. Also, at least asymptotically, there can be no infectives without susceptibles, and if the resistant competitor is an inferior competitor in the absence of infectives, then it cannot survive unless infectives survive as well. The proof is also given in Appendix A.2.

THEOREM 3.2. The following results hold for system (2.1) and when relevant for subsystem (2.2).

1. If $\frac{D_{y}}{\alpha_{y}} \geq S^{0}$, then $\lim _{t \rightarrow \infty} y(t)=0$.

2. If $\frac{D_{y}}{\alpha_{y}}>\frac{D_{I}}{\alpha_{I}}$, then $\lim _{t \rightarrow \infty} y(t)=0$.

3. If $\frac{D_{s}}{\alpha_{s}} \geq S^{0}$, then $\lim _{t \rightarrow \infty} x_{s}(t)=0$.

4. If $\lim _{t \rightarrow \infty} x_{s}(t)=0$, then $\lim _{t \rightarrow \infty} x_{I}(t)=0$, and if also $\frac{D_{y}}{\alpha_{y}}>\frac{D_{s}}{\alpha_{s}}$, then $\lim _{t \rightarrow \infty} y(t)=0$.

5. If $\liminf _{t \rightarrow \infty} x_{s}(t)=0$, then $\liminf _{t \rightarrow \infty} x_{I}(t)=0$.

6. If $\lim _{t \rightarrow \infty} x_{I}(t)=0$ and $\frac{D_{y}}{\alpha_{y}}>\frac{D_{s}}{\alpha_{s}}$, then $\lim _{t \rightarrow \infty} y(t)=0$. 
3.2. Equilibria: Existence, Uniqueness, and Stability. We summarize the criteria for existence, local, and global stability of the equilibria of subsystem (2.2) and of system (2.1) in Table 3.1. The portions of the proofs justifying the results, not given in this section, can be found in Appendix B.

Equilibria of the following form (where the components not indicated explicitly by zero are assumed to be positive) are possible for subsystem (2.2):

$$
E_{0^{*}}=\left(S^{0}, 0,0\right), E_{1^{*}}=\left(\bar{S}, \bar{x}_{s}, 0\right), \text { and } E_{2^{*}}=\left(S^{*}, x_{s}^{*}, x_{I}^{*}\right),
$$

and for the full system (2.1):

$$
\begin{gathered}
E_{0}=\left(S^{0}, 0,0,0\right), E_{1 x}=\left(\bar{S}, \bar{x}_{s}, 0,0\right), E_{1 y}=(\breve{S}, 0,0, \breve{y}) \\
E_{2}=\left(S^{*}, x_{s}^{*}, x_{I}^{*}, 0\right) \text { and } E_{3}=\left(\widehat{S}, \widehat{x}_{s}, \widehat{x}_{I}, \widehat{y}\right)
\end{gathered}
$$

where

$\bar{S}=\frac{D_{s}}{\alpha_{s}}, \quad \bar{x}_{s}=\left(\frac{D \eta_{s}}{D_{s}}\right)\left(S^{0}-\frac{D_{s}}{\alpha_{s}}\right), \quad \breve{S}=\frac{D_{y}}{\alpha_{y}}, \quad \breve{y}=\left(\frac{D \eta_{y}}{D_{y}}\right)\left(S^{0}-\frac{D_{y}}{\alpha_{y}}\right)$

$$
x_{s}^{*}=\frac{D_{I}-\alpha_{I} S^{*}+\gamma}{\delta}, \quad x_{I}^{*}=\frac{x_{s}^{*}\left(-D_{s}+\alpha_{s} S^{*}\right)}{\left(\delta x_{s}^{*}-\gamma\right)},
$$

and $S^{*}$ satisfies

$$
\left(S^{0}-S^{*}\right) D-\frac{\alpha_{s} x_{s}^{*} S^{*}}{\eta_{s}}-\frac{\alpha_{I} x_{I}^{*} S^{*}}{\eta_{I}}=0 \quad \text { with } \quad \frac{D_{s}}{\alpha_{s}}<S^{*}<\frac{D_{I}}{\alpha_{I}} .
$$

Note that once the expressions for $x_{s}^{*}$ and $x_{I}^{*}$ given in (3.2) are substituted into (3.3), this becomes an equation that can be solved for $S^{*}$. Each positive solution $S^{*}$ gives an equilibrium of the form of $E_{2}$ provided the corresponding values of $x_{s}^{*}$ and $x_{I}^{*}$ in (3.2) are positive. This is the case if and only if $\frac{D_{s}}{\alpha_{s}}<S^{*}<\frac{D_{I}}{\alpha_{I}}$, since $x_{s}^{*}>0$ if and only if $S^{*}<\frac{D_{I}+\gamma}{\alpha_{I}}$, and $x_{I}^{*}>0$ if and only if both its numerator and denominator have the same sign. If $S^{*}<\frac{D_{s}}{\alpha_{s}}$, then the denominator is positive, but the numerator is negative. If $S^{*}>\frac{D_{I}}{\alpha_{I}}$, then the numerator is positive, but the denominator is negative. However, both numerator and denominator are positive when $\frac{D_{s}}{\alpha_{s}}<S^{*}<\frac{D_{I}}{\alpha_{I}}$.

$$
\begin{gathered}
\widehat{S}=\frac{D_{y}}{\alpha_{y}}, \quad \widehat{x}_{s}=\left[D_{I}+\gamma-\alpha_{I}\left(\frac{D_{y}}{\alpha_{y}}\right)\right]\left(\frac{1}{\delta}\right), \quad \widehat{x}_{I}=\frac{\widehat{x}_{s}\left(-D_{s}+\alpha_{s} \widehat{S}\right)}{\left(\delta \widehat{x}_{s}-\gamma\right)}, \\
\text { and } \widehat{y}=\left[\left(S^{0}-\widehat{S}\right) D-\frac{\alpha_{s} \widehat{x}_{s} \widehat{S}}{\eta_{s}}-\frac{\alpha_{I} \widehat{x}_{I} \widehat{S}}{\eta_{I}}\right]\left(\frac{\eta_{y}}{\alpha_{y} \widehat{S}}\right) .
\end{gathered}
$$

A similar argument to that just given to ensure that $x_{s}^{*}>0$ and $x_{I}^{*}>0$ can be used to show that both $\widehat{x}_{s}>0$ and $\widehat{x}_{I}>0$ if and only if $\frac{D_{s}}{\alpha_{s}}<\frac{D_{y}}{\alpha_{y}}=\widehat{S}<\frac{D_{I}}{\alpha_{I}}$. The criterion ensuring that $\widehat{y}>0$ is indicated by ${ }^{* *}$ in Table 3.1 . 


\begin{tabular}{|c|c|c|c|}
\hline \multicolumn{4}{|c|}{ Equilibria - Existence and Stability for Subsystem (2.2) } \\
\hline & Existence & Local Asymptotic Stability & Global Asymptotic Stability \\
\hline$E_{0 *}$ & always & $S^{0}<\frac{D_{s}}{\alpha_{s}}$ & no more assumptions needed \\
\hline$E_{1 *}$ & $S^{0}>\frac{D_{s}}{\alpha_{s}}$ & $\mathcal{R}_{0}<1\left(S^{0}<\lambda_{c}\right)$ & $D_{I}$ sufficiently large* \\
\hline$E_{2 *}$ & Theorem 3.4 & Theorem 3.4 & $D=D_{s}=D_{I}, \eta_{s}=\eta_{I}=\eta^{* * *}$ \\
\hline \multicolumn{4}{|c|}{ Equilibria - Existence and Stability for System (2.1) } \\
\hline & Existence & Local Asymptotic Stability & Global Asymptotic Stability \\
\hline$E_{0}$ & always & $S^{0}<\min \left\{\frac{D_{s}}{\alpha_{s}}, \frac{D_{y}}{\alpha_{y}}\right\}$ & no more assumptions needed \\
\hline$E_{1 x}$ & $S^{0}>\frac{D_{s}}{\alpha_{s}}$ & $S^{0}<\lambda_{c} \& \frac{D_{y}}{\alpha_{y}}>\frac{D_{s}}{\alpha_{s}}$ & $D_{I}$ sufficiently large ${ }^{*} \& \frac{D_{y}}{\alpha_{y}}>S^{0}$ \\
\hline$E_{1 y}$ & $S^{0}>\frac{D_{y}}{\alpha_{y}}$ & $\frac{D_{y}}{\alpha_{y}}<\frac{D_{s}}{\alpha_{s}}$ & no more assumptions needed \\
\hline$E_{2}$ & same as $E_{2^{*}}$ & same as $E_{2^{*}} \& S^{*}<\frac{D_{y}}{\alpha_{y}}$ & $\begin{array}{c}E_{2 *} \text { is globally stable for }(2.2) \\
\& \frac{D_{I}}{\alpha_{I}}<\frac{D_{y}}{\alpha_{y}}\end{array}$ \\
\hline$E_{3}$ & $\frac{D_{s}}{\alpha_{s}}<\frac{D_{y}}{\alpha_{y}}<\frac{D_{I}}{\alpha_{I}} \&^{* *}$ & when it exists ${ }^{* * *}$ & unknown \\
\hline $\begin{array}{l}\text { *** on } \\
\text { and ( } 2 . \\
\text { stable } \\
\text { eigenva } \\
\text { the glo } \\
\qquad T \\
\text { The co } \\
\text { siderat } \\
\text { real pa } \\
\text { The co }\end{array}$ & 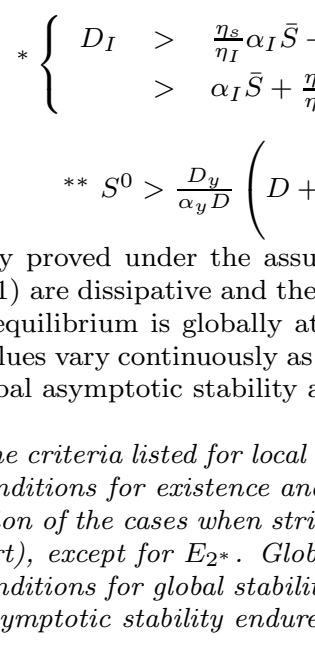 & $\begin{array}{l}\bar{x}_{s}\left(\delta-\frac{\gamma}{S^{0}} \min \left\{\frac{1}{\eta_{s}}, \frac{\alpha_{I}}{\alpha_{s} \eta_{I}}\right\}\right) \\
-\bar{x}_{s}\left(\delta-\frac{\gamma}{S^{0}} \min \left\{\frac{1}{\eta_{s}}, \frac{\alpha_{I}}{\alpha_{s} \eta_{I}}\right\}\right) \\
\frac{\alpha_{s}}{\delta \eta_{I} \eta_{s}}\left(D_{I}+\gamma-\frac{\alpha_{I} D_{y}}{\alpha_{y}}\right)\left(\eta_{I}\right. \\
\text { nption that } \eta_{s}=\eta_{I}=\eta \text { an } \\
\text { equilibria are hyperbolic, the } \\
\text { racting is an open condition } \\
\text { unctions of the parameters, th } \\
\text { lso hold under small perturba } \\
\text { TABLE } 3.1 \\
\text { tability are also required for g } \\
\text { for local stability are both ne } \\
\text { t inequality is replaced by equ } \\
\text { ll stability is with respect to } i \\
y \text { are only sufficient, except in }\end{array}$ & $\begin{array}{l}\quad \text { if } \quad \eta_{s} \geq \eta_{I} \\
\gamma\left(\frac{\eta_{I}}{\eta_{s}}-1\right) \quad \text { if } \quad \eta_{s}<\eta_{I} \\
\left.+\eta_{s}\left(\frac{D_{y}}{\left(\frac{D_{I}}{\alpha_{I}}-\frac{D_{s}}{\alpha_{s}}\right)}\right)\right) \equiv \Psi \\
\left.D=D_{s}\right)=D_{I}=D_{y} \text {. Since (2.2) } \\
\text { roperty that a locally asymptotically } \\
\mathrm{n} \text { parameter space (see [29]). Since } \\
\text { local asymptotic stability and hence } \\
\text { ions of these parameters. } \\
\text { bal stability, and so are not repeated. } \\
\text { essary and sufficient (excluding con- } \\
\text { ity resulting in eigenvalues with zero } \\
\text { itial conditions in the positive cone. } \\
\text { the cases of } E_{0 *}, E_{0}, \text { and } E_{1 y} \text {, where }\end{array}$ \\
\hline
\end{tabular}

The basic reproduction number $\mathcal{R}_{0}$ for both system (2.1) and subsystem (2.2) can be given by

$$
\mathcal{R}_{0} \equiv \frac{\alpha_{I} \bar{S}+\delta \bar{x}_{s}}{D_{I}+\gamma}=\frac{D_{s} \alpha_{I}}{\alpha_{s}\left(D_{I}+\gamma\right)}+\frac{\delta D \eta_{s}\left(S^{0}-\frac{D_{s}}{\alpha_{s}}\right)}{D_{s}\left(D_{I}+\gamma\right)}
$$

The numerator represents the mean number of infectives produced per unit time from both horizontal and vertical transmission resulting from the introduction of a single infective into an otherwise totally susceptible population at equilibrium, and $\frac{1}{D_{I}+\gamma}$ represents the mean amount of time an infective individual remains in the chemostat as an infective. 
We also define the closely related parameter,

$$
\lambda_{c} \equiv \frac{D_{s}}{\delta D \eta_{s}}\left(D_{I}+\gamma+\frac{\delta D \eta_{s}}{\alpha_{s}}-\frac{\alpha_{I} D_{s}}{\alpha_{s}}\right) .
$$

A simple calculation shows that $\mathcal{R}_{0}>1$ is equivalent to $S^{0}>\lambda_{c}$ and $\mathcal{R}_{0}<1$ is equivalent to $S^{0}<\lambda_{c}$. Under our assumptions that $D_{s}<D_{I}$ and $\alpha_{s} \geq \alpha_{I}$, it follows that $\lambda_{c}>\frac{D_{s}}{\alpha_{s}}$.

REMARK 3.3. There is at most one equilibrium of the form $E_{0^{*}}, E_{1^{*}}, E_{0}, E_{1 x}$, $E_{1 y}$, and $E_{3}$. However, there can be up to three equilibria of the form $E_{2^{*}}$. Of course, the number of equilibria of the form $E_{2}$ is always the same as the number of equilibria of the form $E_{2^{*}}$.

The next result addresses the existence, number of equilibria of the form $E_{2 *}$ (and hence $E_{2}$ ), and the stability of $E_{2^{*}}$ for subsystem (2.2).

THEOREM 3.4. Consider subsystem (2.2).

1. Assume that $\mathcal{R}_{0}>1$ or equivalently $S^{0}>\lambda_{c}$.

(i) If $\eta_{s}=\eta_{I}$, then $E_{2^{*}}$ exists, is unique, and is locally asymptotically stable.

(ii) If $\eta_{s}>\eta_{I}$, then $E_{2^{*}}$ exists and is unique. It is locally asymptotically stable if $\frac{\alpha_{s}+\alpha_{I}}{\delta} \geq \eta_{s}-\eta_{I}$. If it loses stability, it can only do so through a Hopf bifurcation.

(iii) If $\eta_{s}<\eta_{I}$, then at least one and at most three equilibria of the form $E_{2^{*}}$ exist. If in addition at least one of the following holds:

$$
\begin{gathered}
D_{I}<\gamma, \\
\eta_{I} \leq \frac{\alpha_{s} \gamma \eta_{s}}{\alpha_{s} D_{I}-\alpha_{I} D_{s}}+\eta_{s},
\end{gathered}
$$

then $E_{2 *}$ is unique and is locally asymptotically stable. If neither (3.7) nor (3.8) holds, saddle node bifurcations are possible. However, no Hopf bifurcation is possible in this case.

2. Assume that $\mathcal{R}_{0}<1$ or equivalently $S^{0}<\lambda_{c}$.

(i) If $\eta_{s} \geq \eta_{I}$, then no equilibrium of the form $E_{2^{*}}$ exists.

(ii) If $\eta_{s}<\eta_{I}$, then at most two equilibria of the form $E_{2^{*}}$ are possible, and generically there is either no equilibrium of this form or there are two. Saddle node bifurcation is possible, but Hopf bifurcation is not.

The proof is given in Appendix B.1.

The condition for local asymptotic stability of $E_{2 *}$ given in Theorem 3.4 part 1.(ii) is only sufficient. It is a simplification of the criterion established in Appendix B.1, using the Routh Hurwicz criterion where it is shown that this equilibrium is locally asymptotically stability when the expression given in (B.6) is positive, and unstable if it is negative. Although positive terms in (B.6) seem to far outnumber the negative terms, this expression can change sign resulting in a Hopf bifurcation as will be illustrated in Figure 3.4.

Example of non-uniqueness of $E_{2^{*}}$ : Set parameters $S^{0}=110, D=0.19, D_{s}=0.2$, $D_{I}=1, \alpha_{s}=0.5, \alpha_{I}=0.4, \eta_{s}=0.01, \eta_{I}=1, \delta=1$, and $\gamma=0.02$. Both criteria (3.7) and (3.8) fail in this case, i.e. $D_{I}=1>0.2=\gamma$ and $\eta_{I}=1>0.01024 \approx$ $\frac{\alpha_{s} \gamma \eta_{s}}{\alpha_{s} D_{I}-\alpha_{I} D_{s}}+\eta_{s}$. By substituting $x_{s}^{*}$ and $x_{I}^{*}$ given by (3.2) into (3.3), we obtain a cubic polynomial in $S$ :

$$
f(S)=-0.0792 S^{3}+0.4024 S^{2}-0.594684 S+0.209,
$$


with roots that give possible values for $S^{*}$. $(f(S)$ is defined explicitly in general in Appendix B.1 equation (B.1).) We are only interested in roots which give an $E_{2^{*}}$ with all components positive. Hence, we only consider roots $S^{*} \in\left(\frac{D_{s}}{\alpha_{s}}, \frac{D_{I}}{\alpha_{I}}\right)$. Solving $f(S)=0$, there are three distinct roots: $0.5094696702,2.074468768$, and 2.496869643 and all three fall in the interval $\left(\frac{D_{s}}{\alpha_{s}}, \frac{D_{I}}{\alpha_{I}}\right)=(0.4,2.5)$, giving 3 distinct equilibria of the form $E_{2^{*}}$ with all components positive. In Figure 3.5 one can see that three equilibria of the form $E_{2^{*}}$ exist for a wide range of the parameter $S^{0}$. Note that two of the three equilibria of the form $E_{2^{*}}$ arise out of a saddle-node bifurcation that will be described in more detail in $\S 3.4$.

We close this subsection with some observations related to the equilibrium $E_{3}$ of system (2.1). The proof is given in Appendix B.3. See also Figure 3.7.

THEOREM 3.5. Assume that an equilibrium of the form $E_{3}$ exists for system (2.1).

1. $E_{0}, E_{1 x}, E_{1 y}$, and at least one equilibrium of the form $E_{2}$ exists.

2. If $\eta_{s} \geq \eta_{I}$, then $S^{*}>\widehat{S}=\frac{D_{y}}{\alpha_{y}}$.

3. If $\eta_{s} \geq \eta_{I}$, then $E_{3}$ is the only equilibrium that can be locally asymptotically stable. If $\eta_{s}<\eta_{I}$, then $E_{1 x}$ or an equilibrium of the form $E_{2}$ can be locally asymptotically stable.

We only obtained conditions for local asymptotic stability of $E_{3}$ in the special case: $\eta_{s}=\eta_{I}=\eta$ and $D=D_{s}=D_{I}=D_{y}$. Restricting the parameters in this way simplified the analysis, since it allowed us to consider a limiting three dimensional system. Since eigenvalues of a matrix vary continuously as functions of the parameters, it follows that local stability of $E_{3}$ still holds at least under small perturbation of the parameters. The example illustrated in Figure 3.1 shows that $E_{3}$ can still be locally asymptotically stable and coexistence of all three populations is still possible even when the difference between the death rates as well as between the yield constants is relatively large. However, if we relax these assumptions it is possible for $E_{3}$ to lose stability through a Hopf bifurcation as will be shown in $\S 3.4 .1$.

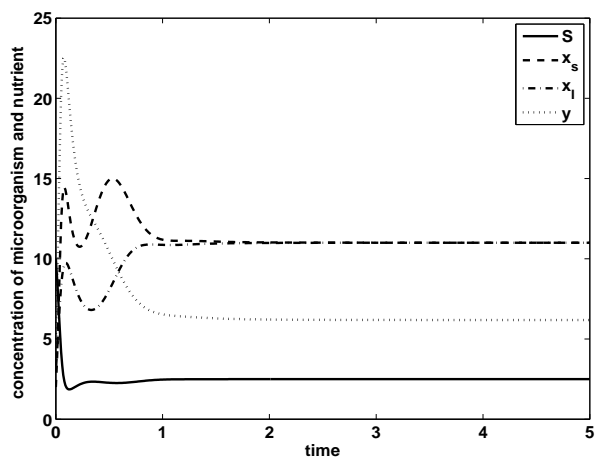

FIG. 3.1. Timeseries illustrating coexistence of all three species in system (2.1). Parameters: $S^{0}=10, D=8, D_{s}=10, D_{I}=20, D_{y}=15, \alpha_{s}=7, \alpha_{I}=5, \alpha_{y}=6, \eta_{s}=10, \eta_{I}=5, \eta_{y}=7$, $\delta=0.7$, and $\gamma=0.2$. Initial conditions: $\left(S(0), x_{s}(0), x_{I}(0), y(0)\right)=(10,2,3,5) . \quad E_{3}$ is locally asymptotically stable, even though the difference between the death rates and the yield constants is relatively large.

REMARK 3.6. The results on existence, and local and global stability of the various equilibria in this model are summarized in Table 3.1 and proved in Appendix B. 
3.3. Criteria for Persistence of the Populations. Some related notation and preliminary results are given in Appendix A.1. The proofs of the results in this section for subsystem (2.2) can be found in Appendix C.

DEFINITION 3.7. A population $z(t)$ is said to be uniformly strongly persistent (uniformly weakly persistent) if there exists $\epsilon>0$, such that $\liminf _{t \rightarrow \infty} z(t) \geq \epsilon$ $\left(\limsup _{t \rightarrow \infty} z(t) \geq \epsilon\right)$, where $\epsilon$ is independent of the initial conditions, provided $z(0)>$ 0 .

THEOREM 3.8. The following hold for system (2.1) and when relevant for subsystem (2.2).

1. If $\frac{D_{s}}{\alpha_{s}}>\frac{D_{y}}{\alpha_{y}}$, then $\lim _{t \rightarrow \infty}\left(x_{s}+x_{I}\right)(t)=0$. If in addition, $S^{0}>\frac{D_{y}}{\alpha_{y}}$, then $E_{1 y}$ is globally asymptotically stable with respect to solutions with $y(0)>0$.

2. If $x_{I}(t)$ is uniformly strongly persistent, then $x_{s}(t)$ is uniformly strongly persistent.

3. If $\left(x_{s}+x_{I}\right)(t)$ is strongly persistent, then $x_{s}(t)$ is strongly persistent. If $\left(x_{s}+x_{I}\right)(t)$ is uniformly strongly persistent, then $x_{s}(t)$ is uniformly strongly persistent.

4. If $x_{I}(t)$ is weakly persistent and $S^{0}<\frac{D_{I}+\gamma}{\alpha_{I}}$, then $x_{s}(t)$ is uniformly strongly persistent.

5. If $S^{0}>\frac{D_{s}}{\alpha_{s}}$, then $x_{s}(t)$ is uniformly strongly persistent for subsystem (2.2). If in addition, $\frac{D_{y}}{\alpha_{y}}>\frac{D_{s}}{\alpha_{s}}$, then $x_{s}(t)$ is also uniformly strongly persistent for system 2.1).

6. If $R_{0}>1$ (or equivalently $S^{0}>\lambda_{c}$ ), then both $x_{s}(t)$ and $x_{I}(t)$ are uniformly strongly persistent for subsystem (2.2). If in addition, $\frac{D_{y}}{\alpha_{y}}>\frac{D_{s}}{\alpha_{s}}$, and $x_{I}(0)>0$ implies that $\lim _{t \rightarrow \infty} y(t)=0$, then both $x_{s}(t)$ and $x_{I}(t)$ are also uniformly strongly persistent for system (2.1).

7. If $R_{0}>1, E_{2^{*}}$ is globally asymptotically stable for subsystem (2.2), and $S^{*}>\frac{D_{y}}{\alpha_{y}}>\frac{D_{s}}{\alpha_{s}}$, then all three populations $x_{s}(t), x_{I}(t)$, and $y(t)$ are uniformly strongly persistent, and so system (2.1) is uniformly strongly persistent.

REMARK 3.9.

1. Using Theorem 3.2 parts 1. and 6., it also follows that when $\frac{D_{y}}{\alpha_{y}}>\frac{S_{s}}{\alpha_{s}}$, if $y(t)$ is weakly perisistent, then $x_{I}(t)$ is weakly persistent.

2. Theorem 3.8 combined with the global stability results in Table 3.1 basically says that for subsystem (2.2), the persistence of the infected and susceptible populations depends upon $S^{0}$, the input concentration of the nutrient. If it is too low, neither population survives. For intermediate values $x_{s}(t)$ persists, and whether or not $x_{I}$ survives can also depend upon the relative values of the yield constants. For high enough concentrations both populations persist. As well, when the infection persists, there will always be susceptibles, since $x_{I}(t)$ cannot persist unless $x_{s}(t)$ also persists. Using Theorem 3.2 parts 1. and 6., it also follows that when $\frac{D_{y}}{\alpha_{y}}>\frac{S_{s}}{\alpha_{s}}$, competitor $y(t)$ can only survive if some infectives survive as well.

3. From Theorem 3.2 2. and Theorem 3.8 part 1. it follows that for both the infected population and the resistant population to persist, $\frac{D_{s}}{\alpha_{s}} \leq \frac{D_{y}}{\alpha_{y}} \leq \frac{D_{I}}{\alpha_{I}}$ must hold, i.e., the resistant population y must be a less effective competitor than the susceptible population $x_{s}$, but a more effective competitor than the infected population $x_{I}$. This is also a necessary condition for a coexistence steady to exist as indicated in Table 3.1.

4. It is surprising to note that even if $S^{0}<\frac{D_{I}+\gamma}{\alpha_{I}}$, it is possible for $x_{I}$ to survive. In particular, one can show that $\lambda_{c}<S^{0}<\frac{D_{I}+\gamma}{\alpha_{I}}$ if and only if $D_{s} \alpha_{I}<\delta D \eta_{s}$. In fact, in the numerical simulation illustrated in Figure 3.2, $S^{0}<\frac{D_{I}}{\alpha_{I}}$ and yet all three 
populations survive. For all of the initial conditions we tried, there was convergence to the coexistence equilibrium $E_{3}$, and so it is likely that $E_{3}$ is globally asymptotically stable, and hence all populations are uniformly strongly persistent in this example.

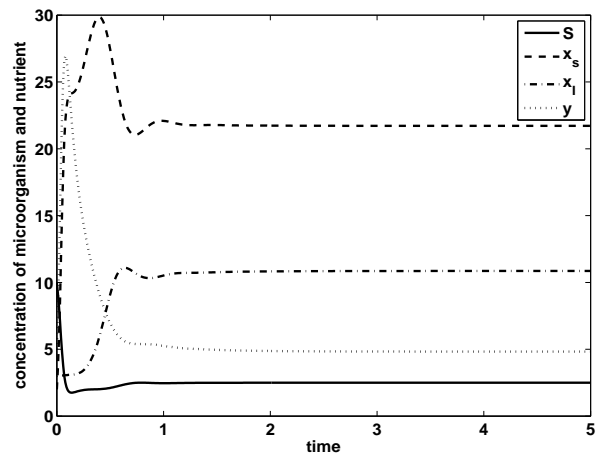

FIG. 3.2. Timeseries illustrating coexistence of all three species in system (2.1), and hence $x_{I}$, even though $S^{0}<\frac{D_{I}}{\alpha_{I}}$. Parameters: $S^{0}=9.9$ and $\alpha_{I}=2$. All of the other parameters are the same as for Figure 3.1. Initial conditions: $\left(S(0), x_{s}(0), x_{I}(0), y(0)\right)=(10,2,3,5)$.

3.4. Bifurcation Analysis. System (2.1) and subsystem (2.2) have rich dynamics. Equilibria are involved in transcritical, saddle-node, Hopf, and homoclinic bifurcations. As well it is possible to have a saddle-node bifurcation of limit cycles. Multiple stable attractors are possible, and there can be more than one periodic orbit. We illustrate some of these interesting phenomena by means of bifurcation diagrams. All bifurcation diagrams included were created using XPPAUT [13] and MATLAB [18]. In all of these diagrams, a solid/dashed line represents a stable/unstable equilibrium, and a closed/open circle represents a stable/unstable periodic orbit. We consider the cases $\eta_{s} \geq \eta_{I}$ and $\eta_{s}<\eta_{I}$ separately, since different bifurcations are possible in each of these cases. We also assume that $\frac{D_{s}}{\alpha_{s}}<\frac{D_{y}}{\alpha_{y}}$, since otherwise both $x_{s}$ and $x_{I}$ die out (see Theorem 3.8 part 1.). Parameters are chosen for illustrative purposes only, and not based on experimental data.

3.4.1. Case 1: $\eta_{s} \geq \eta_{I}$. Recall that there is at most one equilibrium of the form $E_{2}$ in this case, and since there is always at most one equilibrium of any of the other forms, no saddle-node bifurcation of equilibria is possible.

\section{Transcritical Bifurcation of Equilibria}

In this case, in system (2.1) there is typically a successive transfer of stability from $E_{0}$ to $E_{1 x}$ to $E_{2}$ to $E_{3}$, each transfer via a transcritical bifurcation, as the parameter $S^{0}$ is increased. For an example see Figure 3.3. For $S^{0} \in\left(0, \frac{D_{s}}{\alpha_{s}}\right), E_{0}$ is stable. $E_{0}$ coalesces with and transfers stability to $E_{1 x}$ when $S^{0}=\frac{D_{s}}{\alpha_{s}}$. For $S^{0} \in\left(\frac{D_{s}}{\alpha_{s}}, \lambda_{c}\right), E_{1 x}$ is stable. $E_{1 x}$ coalsces with $E_{2}$ when $S^{0}=\lambda_{c}$, and then stability is transferred to $E_{2}$. For $S^{0} \in\left(\lambda_{c}, \Psi\right), E_{2}$ is stable (where $\Psi$ was defined when the existence of $E_{3}$ was addressed in Table 3.1). $E_{2}$ coalsces with $E_{3}$ when $S^{0}=\Psi$ and then stability is transferred to $E_{3}$.

Hopf Bifurcation of $E_{2^{*}}$ and $E_{2}$, and Saddle Node Bifurcation of Limit Cycles 

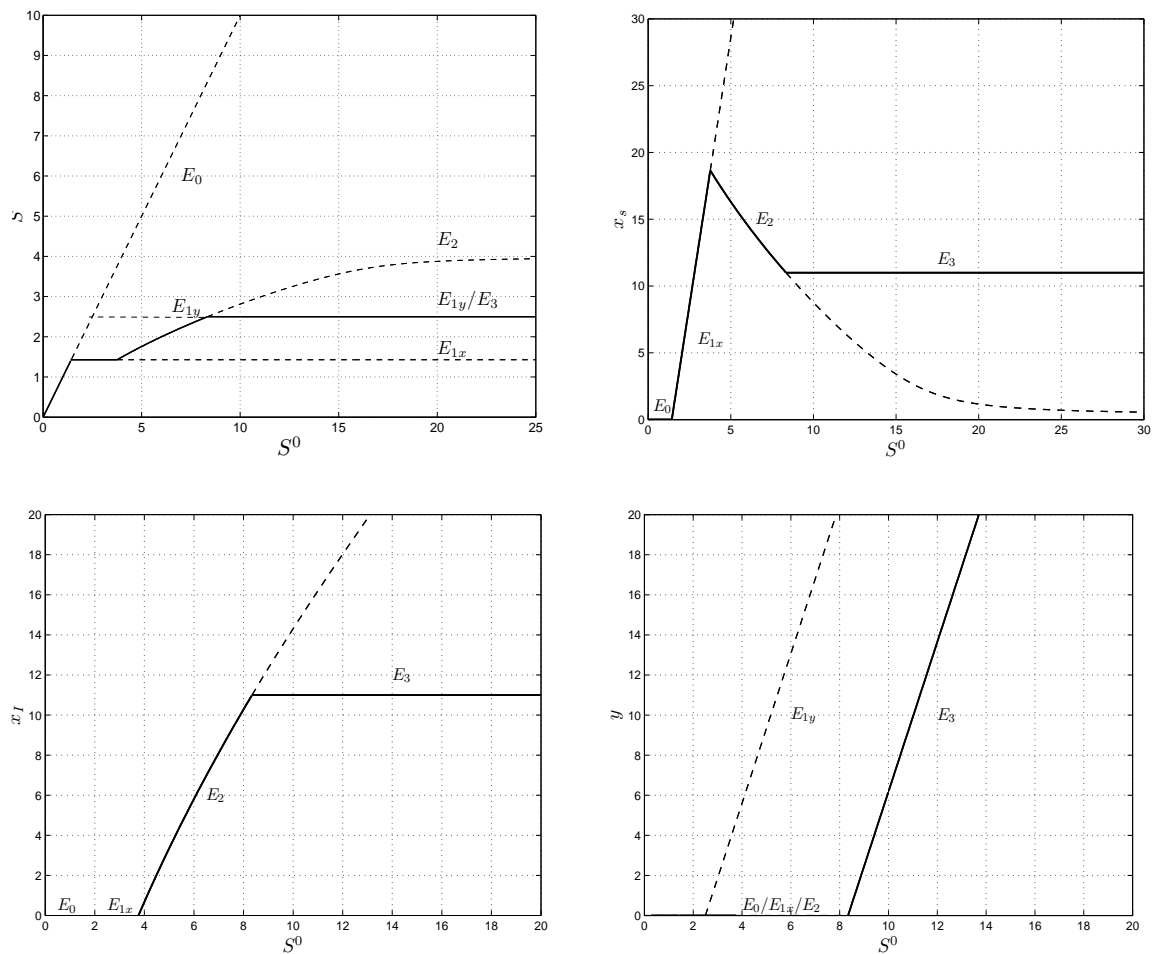

Fig. 3.3. Bifurcation diagrams for system (2.1) with $\eta_{s}>\eta_{I}$. Parameters: $D=8, D_{s}=10$, $D_{I}=20, D_{y}=15, \alpha_{s}=7, \alpha_{I}=5, \alpha_{y}=6, \eta_{s}=10, \eta_{I}=5, \eta_{y}=7, \delta=0.7$, and $\gamma=0.2$. The top left and right graphs have $S$ and $x_{s}$ on the ordinate axis, respectively. Both show the series of bifurcations that occur where stability is transferred successively from $E_{0}$ to $E_{1 x}$ at $S^{0}=\frac{D_{s}}{\alpha_{s}} \approx 1.43$, to $E_{2}$ at $S^{0}=\lambda_{c} \approx 3.76$, and then to $E_{3}$ at $S^{0}=\Psi \approx 8.34$, all via transcritical bifurcations. There is also a transcritical bifurcation involving $E_{0}$ and $E_{1 y}$, shown in the top left graph. However, both equilibria are unstable. The bottom left and right graphs have $x_{I}$ and $y$ on the ordinate axis, respectively, and show the transfer of stability from $E_{2}$ to $E_{3}$. The bottom right graph shows that $E_{1 y}$ and $E_{3}$ are not involved in a bifurcation at $S^{0}=\Psi$, even though the curves meet and even overlap, since the $S$ components are equal in the top left graph. $E_{1 y}$ remains unstable.

In this case, the equilibrium $E_{2}$ in system (2.1) ( $E_{2^{*}}$ for subsystem $(2.2)$ ) can be involved in a Hopf bifurcation. We only gave sufficient conditions for the local asymptotic stability of $E_{2^{*}}$ in Theorem 3.4 part 1.(ii), based on the signs of the expressions given in (B.5) and (B.6). Hopf bifurcation is possible if $\eta_{s}>\eta_{I}$, since (B.6) can change sign from positive to negative as $\eta_{s}$ increases beyond $\eta_{I}$. As this bifurcation occurs in the face where $y \equiv 0$, we illustrate it for subsystem (2.2) in Figure 3.4. As the parameter $\eta_{s}$ is increased from zero, there is a saddle-node bifurcation of limit cycles resulting in two periodic orbits, one orbitally asymptotically stable and one unstable. As $\eta_{s}$ is increased further, the unstable periodic orbit disappears in a subcritical Hopf bifurcation at $E_{2^{*}}$.

3.4.2. Case 2: $\eta_{s}<\eta_{I}$. Non-uniqueness of equilibria of the form $E_{2}$ is possible in this case (see Theorem 3.4 parts 1.(ii) and 2.(ii)). However, unlike in the previous case, no Hopf bifurcation involving $E_{2^{*}}$ or $E_{2}$ is possible. 

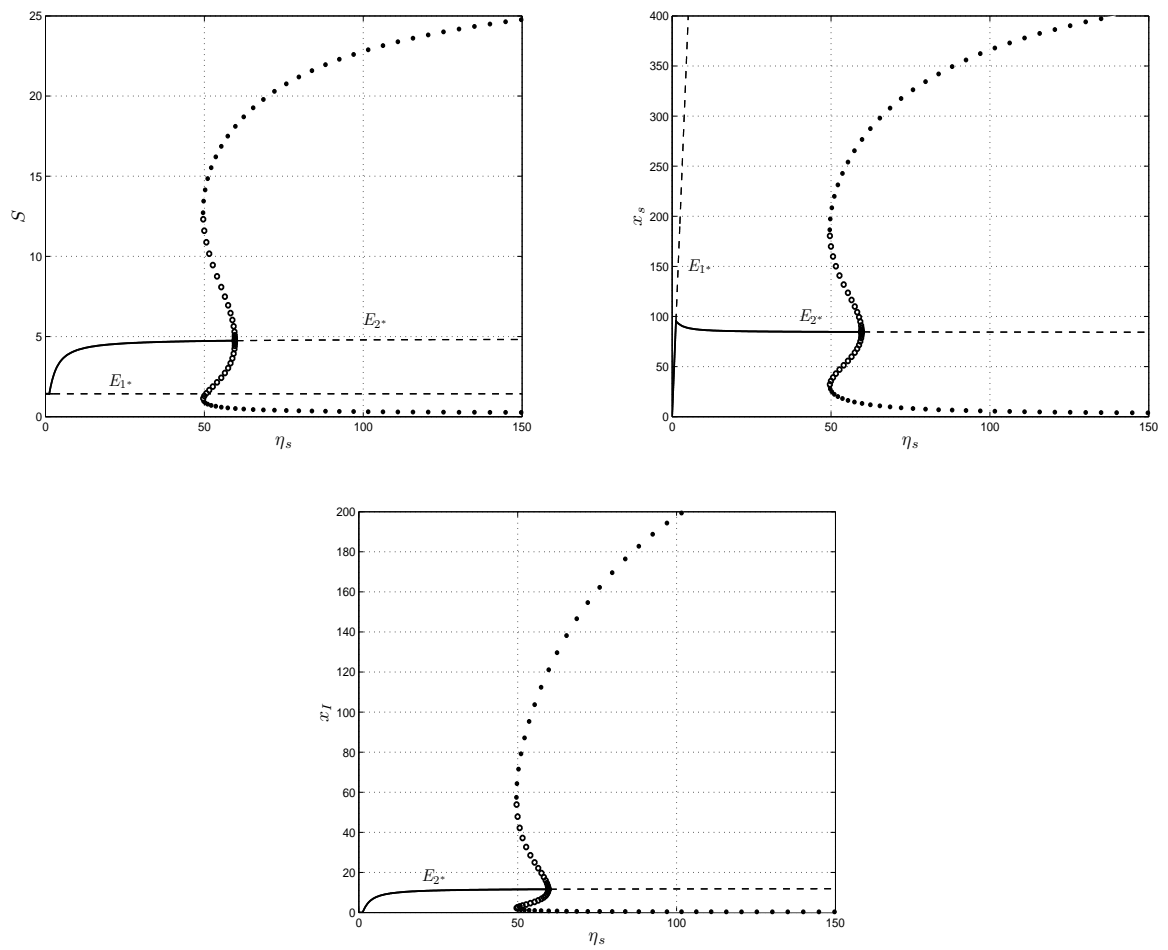

FIG. 3.4. A saddle-node bifurcation of limit cycles and a subcritical Hopf bifurcation of $E_{2 *}$ as $\eta_{s}$ is varied in subsystem (2.2). Parameters: $S^{0}=100, D=8, D_{s}=10, D_{I}=200, \alpha_{s}=7$, $\alpha_{I}=6.5, \eta_{I}=0.5, \delta=2$, and $\gamma=0.01$. There is a range of the parameter $\eta_{s}$ for which there are two limit cycles, the one with larger amplitude orbitally asymptotically stable and the other one unstable. A Hopf bifurcation of $E_{2^{*}}$ is not possible unless $\eta_{s}>\eta_{I}$.

Transcritical Bifurcations and Saddle-Node Bifurcation of $E_{2^{*}}$ and $E_{2}$

The sequence of transcritical bifurcations that occur as $S^{0}$ is varied is the same as in the case of $\eta_{s} \geq \eta_{I}$. Compare Figure 3.3 with Figures 3.6 and 3.7. If also (3.7) or (3.8) holds, then no saddle-node bifurcation of equilibria is possible either. However, when both of these conditions are violated, $E_{2^{*}}$ and hence $E_{2}$ can be involved in two saddle-node bifurcations (see Figure 3.5 for subsystem (2.2) and Figure 3.6 for system (2.1)). Notice that in this example, when this occurs and hence three equilibria of the form $E_{2^{*}}$ exist for subsystem (2.2), two are asymptotically stable and one is unstable. However, when species $y$ is introduced to obtain system (2.1), one of the stable equilibria becomes unstable. These saddle-node bifurcations are also illustrated in the example shown in Figure 3.7.

\section{Hopf Bifurcation of $E_{3}$ and Homoclinic Bifurcation}

When $\eta_{I}>\eta_{s}$ it is not possible for the equilibrium $E_{2}$ in system (2.1) or $E_{2^{*}}$ for subsystem (2.2) to be involved in a Hopf bifurcation. However, it is possible for $E_{3}$ to undergo a Hopf bifurcation. Figure 3.6 illustrates this Hopf bifurcation for system (2.1). The period of the periodic orbit increases as the parameter $S^{0}$ is decreased. In fact, it appears that as $S^{0}$ approaches a critical value, around $S^{0}=173.9$ in the bifurcation diagram in Figure 3.6, the period of the periodic orbit approaches infinity. 

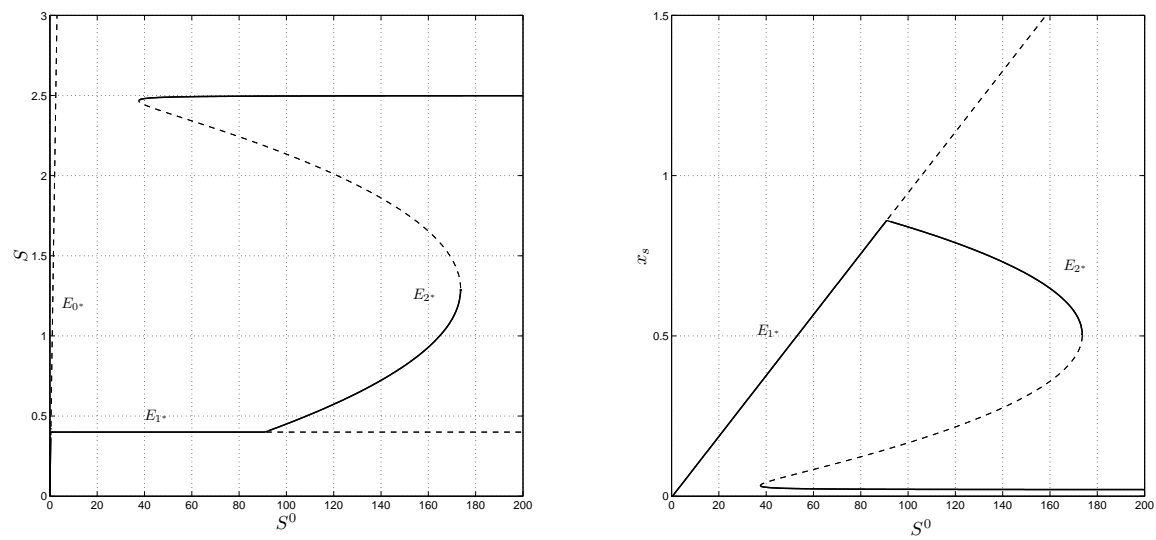

Fig. 3.5. Bifurcation diagrams for subsystem (2.2) $\eta_{I}>\eta_{s}$ as $S^{0}$ is varied. Parameters: $D=0.19, D_{s}=0.2, D_{I}=1, \alpha_{s}=0.5, \alpha_{I}=0.4, \eta_{s}=0.01, \eta_{I}=1, \delta=1$, and $\gamma=0.02$. Besides the transcritical bifurcations involving $E_{0^{*}}$ and $E_{1^{*}}$ and $E_{1^{*}}$ and $E_{2^{*}}$, both graphs show two saddlenode bifurcations of $E_{2^{*}}$. Thus there is a parameter range for which there are three equilibria of the form $E_{2^{*}}$. Two of these can be stable for the same value of $S^{0}$ or one equilibrium of the form of $E_{2 *}$ and $E_{1 *}$ can both be stable, demonstrating the possibility of a hysteresis effect in both cases.

Hence, it seems that the periodic orbits originate from a homoclinic bifurcation that involves one of the $E_{2}$ equilibria that is a saddle point.

The model predicts possible coexistence of all three species once $S^{0}$ is above the critical value for the homoclinic bifurcation. However, in this example, if $S^{0}$ is not large enough, the amplitude of the oscillations of the $x_{s} x_{I}$, and $y$ populations would be very large, with their lowest values getting dangerously close to 0 , so that a stochastic event could easily wipe out one or more of the populations. This seems less likely as $S^{0}$ becomes sufficiently large. Once $S^{0}$ increases past the critical value for the Hopf bifurcation, the coexistence of $x_{s}, x_{I}$ and $y$ seems more likely.

A similar sequence of bifurcations is illustrated in Figure 3.7. However, there it is shown that bi-stability and hence initial condition dependent outcomes is possible for a range of the parameter $S^{0}$.

4. Discussion. In this paper the effect of a virus on two competing populations, one susceptible and the other resistant, was investigated using an SIS epidemic model in a chemostat-like environment. As expected the basic reproduction number, given in (3.2), played an important role in predicting which populations survive (see Table 3.1) as did $S^{0}$ the concentration in the input reservoir. That these two quantities are related is apparent, since we showed that $\mathcal{R}_{0}>1$ is equivalent to $S^{0}>\lambda_{c}$ where $\lambda_{c}$ was defined in (3.6).

It was shown that although competitive exclusion occurs when there is no virus, coexistence of competitors is possible in the presence of the virus. However, this is only possible if $D_{s} / \alpha_{s}<D_{y} / \alpha_{y}<D_{I} / \alpha_{I}$, i.e., the resistant population if an intermediate competitor in the absence of the virus. On the other hand, surprisingly, it is not necessary for $D_{I} / \alpha_{I}<S^{0}$ for the survival of all three populations to coexist. Our results predict that the presence of viruses in the ocean may enhanced bacterial diversity. They therefore also predict that elimination of a virus could result in the extinction of bacterial species. In an extreme case, an attempt to eliminate a virus 

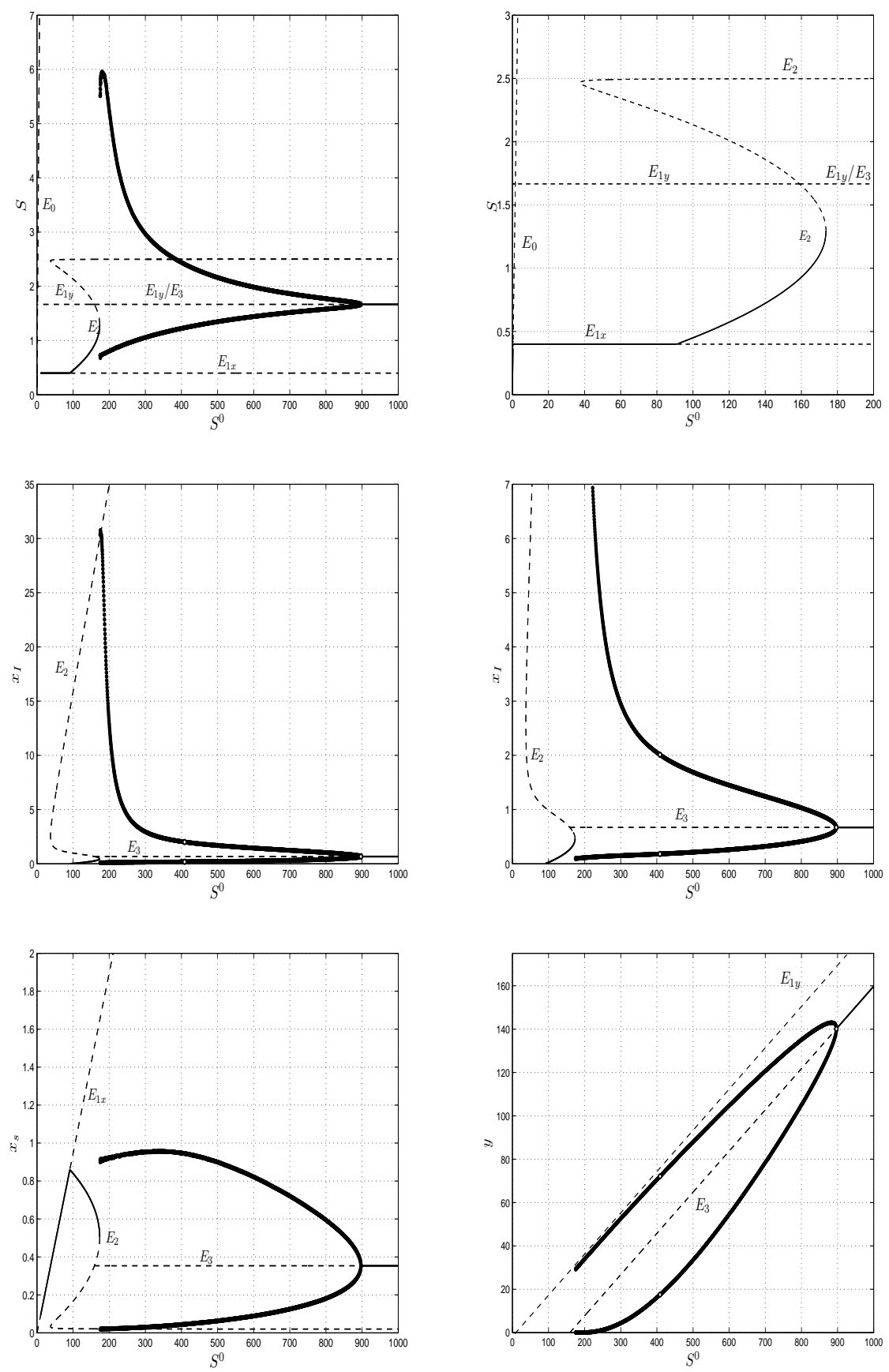

FIG. 3.6. Bifurcation diagrams for system (2.1) with $\eta_{I}>\eta_{s}$ as $S^{0}$ varies, illustrating the effect of introducing $y$ to the example illustrated in Figure 3.5. Parameters: $\alpha_{y}=0.6$ and $D_{y}=1$. All the other parameters are the same as for Figure 3.5. The transcritical and saddle-node bifurcations of the equilibria are the same as in Figure 3.5. However, comparing the two top graphs and the lower left one with the graphs in Figure 3.5 shows that one of the equilibria of the form $E_{2 *}$ that was stable for subsystem (2.2) becomes unstable when $y$ is introduced. As $S^{0}$ is increased, a stable periodic orbit is born from a homoclinic bifurcation involving the $E_{2}$ that changed stability when $y$ was introduced. This periodic orbit disappears in a Hopf bifurcation of $E_{3}$. 


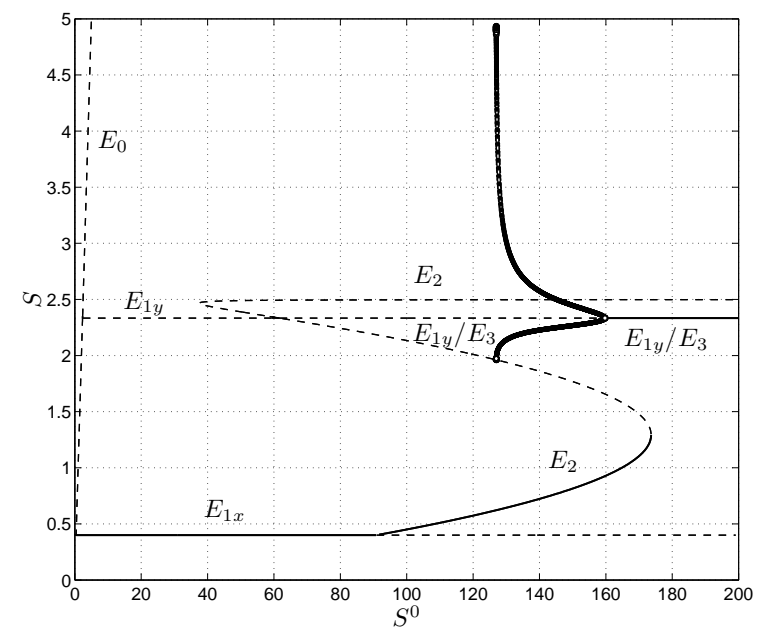

FIG. 3.7. Bifurcation diagram for system (2.1) with $\eta_{I}>\eta_{s}$ as $S^{0}$ varies. Parameters: $D=$ $0.19, D_{s}=0.2, D_{I}=1, D_{y}=1.4, \alpha_{s}=0.5, \alpha_{I}=0.4, \alpha_{y}=0.6, \eta_{s}=0.01, \eta_{I}=1, \eta_{y}=1$, $\delta=1$, and $\gamma=0.02$. The graph shows four transcritical bifurcations involving $E_{0}$ and $E_{1 x}, E_{0}$ and $E_{1 y}, E_{1 x}$ and $E_{2}$, and $E_{2}$ and $E_{3}$; two saddle-node bifurcations involving $E_{2}$; a supercritical Hopf bifurcation of $E_{3}$; and a homoclinic bifurcation involving one of the unstable (saddle point) equilibria of the form $E_{2}$. As $S^{0}$ increases, a stable periodic orbit is born as the homoclinic orbit breaks and then vanishes at the Hopf bifurcation of $E_{3}$. There is bi-stability for a range of values of $S^{0}$, and hence initial condition dependent outcomes, i.e., $E_{2}$ and either $E_{3}$ or the periodic orbit are both stable. Also, there is a range of values of $S^{0}$ where $E_{3}$ exists and is unstable, and either $E_{1 x}$ or $E_{2}$ is locally asymptotically stable.

could end in creating a super-competitor that out-competes many other species. We also showed that in the presence of virus, a rich variety of dynamics is possible including bi-stability and multiple limit cycles. We assumed that population $y$ is completely resistant to the virus. This can be considered a limiting case, and the predictions of this model are likely to hold if $y$ is also susceptible, but the effect of the virus on $y$ is sufficiently small compared to its effect on population $x_{s}$.

In the Lotka-Volterra competition model with SI disease studied by van den Driessche and Zeeman [34], both disease induced oscillatory behavior as well as competition induced oscillatory behavior were shown to occur. Both behaviors also occur in our model as well. However, disease induced oscillatory behavior is likely only to occur in our subsystem (2.2) if $\mathcal{R}_{0}>1$ and $\left(\alpha_{s}+\alpha_{I}\right) / \delta<\eta_{s}-\eta_{I}$ (see Theorem 3.4). This is demonstrated in Figure 3.4. If $\eta_{s}$ is sufficiently large, the periodic solution appears to be globally attracting. For intermediate values of $\eta_{s}$ with $\left(\alpha_{s}+\alpha_{I}\right) / \delta<\eta_{s}-\eta_{I}$, there is bi-stability, and although there is a stable endemic equilibrium and both a stable and an unstable limit cycle, invasion by a small number of resistant bacteria would likely result in coexistence at the stable endemic equilibrium. That the yield constants play a role in the dynamics is interesting here, since in the absence of disease, yield constants can be scaled out of the basic chemostat model, and play no role in determining the outcomes. That invasion by a resistant competitor can induce oscillations when there is no oscillatory behavior when the disease is endemic and there are no resistant bacteria can be observed in the example illustrated in Figures 3.5 and 3.6. Again the yield constants play a role. When $D_{s}=D_{I}=D_{y}$ and $\eta_{s}=\eta_{I}$ it was shown 
in Appendix B that when the coexistence equilibrium $E_{3}$ exists is is always locally asymptotically stable. However, in the example in Figure 3.6, competitor induced oscillatory behavior resulting from a supercritical Hopf bifurcation is observed when $\eta_{I}>\eta_{s}$.

Both the subsystem without the competitor population $y,(2.2)$ and the full system (2.1) exhibit the possibility of a hysteresis effect (see Figures 3.5 and 3.7), respectively. For the parameter values used in Figure 3.5, for small input concentrations of $S^{0}>$ $\frac{D_{s}}{\alpha_{S}}$, the subsystem has only one stable equilibrium and it is of the form $E_{1^{*}}$. As $S^{0}$ increases there is a saddle-node bifurcation of $E_{2^{*}}$ and then the subsystem has two stable equilibria, one of the form $E_{1^{*}}$ and one of the form $E_{2^{*}}$, and the one of the form $E_{1 *}$ has a much higher concentration of susceptibles. As $S^{0}$ increase even further, there is a transcritical bifurcation involving $E_{1^{*}}$ and $E_{2^{*}}$, and above this value there are two stable equilibria for the form $E_{2^{*}}$, with a very low concentration of susceptibles. If the system was first observed at moderate values of $S^{0}$ (between above the transcritical bifurcation but below the saddle-node bifurcation) where there were two stable equilibria of the form $E_{2^{*}}$ and had approached the equilibrium with the larger concentration of susceptibles, if $S^{0}$ was increased past the saddle-node bifurcation, there would be a drastic reduction in the concentration of the susceptible population that could not be corrected by simply lowering $S^{0}$ past the saddle-node bifurcation. One would have to lower $S^{0}$ drastically, beyond the lower saddle-node bifurcation, wait for the population to approach the equilibrium of the form $E_{1^{*}}$, and then start increasing $S^{0}$ back to the intermediate values where the concentration of the susceptible population was healthier. However, this might prove disastrous for a natural habitat where it might not be possible to reduce $S^{0}$ enough to effect the recovery. Similar scenarios could be described for the full system.

Predictions based on the analysis could have other interesting implications for ecological systems. For example, as seen in Figure 3.4, as the parameter $\eta_{s}$ is increased, the amplitude of the oscillations in the population sizes increases. This is pausible, since the size of $\eta_{s}$ relates to the ability of the microorganisms to convert nutrient to biomass, and the better the nutrient-converter, one might expect the higher the maximum possible population size. However, in addition to having a larger maximum population size, the increase in $\eta_{s}$ also results in a smaller minimum population size. Although deterministically the population would not die out, this makes the population more vulnerable to a stochastic event such as a heat or cold spell that could easily result in the extinction of the species if it occurs at one of its low points in the oscillation. Hence, efficiency in conversion of the nutrient may not be an entirely good thing.

Predictions of our model also have implications for the dynamics of conjugationally transmitted plasmids. As mentioned in $\S 2$, subsystem (2.2) was studied (with different notation) in this context. In the special case that (in our notation) $D=D_{s}=D_{I}$ and $\eta_{s} \approx \eta_{I}$ Stewart and Levine [30] argued that the plasmid bearing and plasmid free bacteria can coexist, and when they do coexist there is a unique locally asymptotically stable equilibrium. Assuming that $D=D_{s}=D_{I}$ and $\eta_{s}=\eta_{I}$, Imran and Smith [17] proved that this equilibrium is globally asymptotically stable. However, we showed that although $\left(\alpha_{s}+\alpha_{I}\right) / \delta>\eta_{s}-\eta_{I}$, is a sufficient condition for the local asymptotic stability of this coexistence equilibrium of subsystem (2.2), $\left(E_{2^{*}}\right)$, analysis predicts that this equilibrium can lose stability through a Hopf bifurcation, and that a saddle-node bifurcation of limit cycles can also occur resulting in more than one limit cycle (see Figure 3.4). Thus bi-stability is possible, result- 
ing in sustained oscillatory behavior or convergence to $E_{2^{*}}$, depending on the initial conditions, and hence there can be plasmid induced oscillatory behavior.

It was mentioned earlier (in §2) that an SI epidemic/chemostat model had already been analyzed with a different interpretation. Interestingly, the context/application had nothing to do with epidemiology; it was a model of a food web with a predator feeding on two trophic levels [12], [37]. Many models have many different interpretations. For instance, the model in [22] could also be viewed as a model for predator-prey interactions in the chemostat. As such, our model could also describe various other applications. Although chemostats are typically used to study bacteria, our system could be seen as modelling a fish population in a lake, where fish are competing exploitatively for their food, and where one species is infected by a disease. Within the lake/ocean context, there could be other similar scenarios of interest to a lake or marine ecologist for which this model would apply.

On the other hand, we can make predictions about the outcomes of an SI disease model in the chemostat from the results in [12] and [37]. The only difference between that model and system 2.1 is that in our model we would have to take $\gamma=0$ to obtain their model. However, this apparently small change has important implications for the possible dynamics. In particular, in [37] it is shown that in the SI model there is an extra equilibrium point in which there are infected individuals, but neither susceptibles nor resistant individuals, and in their Theorem 2.1 that it is possible for this equilibrium to be globally asymptotically stable. Therefore, their results can be used to show that it is possible for the infected population to persist in the absence of susceptibles. Their results can also be used to show that is possible that the introduction of infected individuals can result in the entire population becoming infected in the absence of resistant individuals, where as in their presence all three populations can persist. Hence there is competition-mediated coexistence. This is different than for the SIS model that we studied. Recall that in system (2.1) if there are infected individuals there must also always be susceptible individuals and if there are resistant individuals there must also be infected individuals and hence susceptible individuals (see Theorem 3.2 parts 4 . and 6).

Another possible application of our analysis is to wastewater treatment or biological remediation. Our results suggest that new methods could be developed that take advantage of multiple species of bacteria which might be enabled to coexist through the addition of a virus. There is also some renewed interest in studying the use of phage in the treatment of bacterial infections in humans [36], due to the increase in antibiotic-resistant bacteria and our results may find relevance in that context.

\section{APPENDIX}

Throughout the appendix we assume that $D_{s}<D_{I}$ and $\alpha_{s} \geq \alpha_{I}$, unless stated otherwise.

\section{Appendix A. Preliminaries.}

A.1. Useful Notation and Lemmas. For a real-valued function $p$ on $[0, \infty)$ we define

$$
p_{\infty}=\liminf _{t \rightarrow \infty} p(t), \quad p^{\infty}=\limsup _{t \rightarrow \infty} p(t) .
$$

The following Lemma is given in [33] as Corollary 2.4 (a).

Lemma A.1. Let $\mathcal{J}$ be a bounded interval in $\mathbb{R}$ and $g:\left(t_{0}, \infty\right) \times \mathcal{J} \rightarrow$ be bounded and uniformly continuous. Further let $x:\left(t_{0}, \infty\right) \rightarrow \mathcal{J}$ be a solution of 
$x^{\prime}=g(t, x)$, which is defined on the whole interval $\left(t_{0}, \infty\right)$. Then $\liminf _{t \rightarrow \infty} g\left(t, x_{\infty}\right) \leq$ $0 \leq \limsup _{t \rightarrow \infty} g\left(t, x_{\infty}\right)$ and $\liminf _{t \rightarrow \infty} g\left(t, x^{\infty}\right) \leq 0 \leq \limsup _{t \rightarrow \infty} g\left(t, x^{\infty}\right)$. We will also use the following results from [33] given there as Theorem 1.3 and Theorem 4.5.

TheOrem A.2. Let $X$ be a locally compact metric space that is the disjoint union of two sets $X_{1}$ and $X_{2}$ with $X_{2}$ compact in $X$. Let $X_{1}$ be forward invariant under the continuous semiflow $\Phi$ on $X$.

1. Then $X_{2}$ is a uniform strong repeller for $X_{1}$, whenever it is a uniform weak repeller for $X_{1}$.

2. Assume that $\Omega_{2}$,

$$
\Omega_{2}=\cup_{y \in Y_{2}} \omega(y), \quad Y_{2}=\left\{x \in X_{2}: \Phi_{t}(x) \in X_{2}, \forall t>0\right\},
$$

(where $\omega(y)$ denotes the omega limit set of $\Phi_{t}(y)$ ) has an acyclic isolated covering $M=\cup_{k=1}^{m} M_{k}$. If each part $M_{k}$ of $M$ is a weak repeller for $X_{1}$, then $X_{2}$ is a uniform strong repeller for $X_{1}$.

A.2. Proofs of Preliminary Results. Lemma A.3. For all solutions of (2.1) or (2.2), $S_{\infty} \geq \min \left\{\frac{A D}{\alpha B}, S^{0}\right\}>0$, where for system (2.1) $A=\min \left\{\frac{\alpha_{s}}{\eta_{s}}, \frac{\alpha_{I}}{\eta_{I}}, \frac{\alpha_{y}}{\eta_{y}}\right\}, B=$ $\max \left\{\frac{\alpha_{s}}{\eta_{s}}, \frac{\alpha_{I}}{\eta_{I}}, \frac{\alpha_{y}}{\eta_{y}}\right\}$, and $\alpha=\max \left\{\alpha_{s}, \alpha_{y}\right\}$, but for subsystem (2.2) $A=\min \left\{\frac{\alpha_{s}}{\eta_{s}}, \frac{\alpha_{I}}{\eta_{I}}\right\}$, $B=\max \left\{\frac{\alpha_{s}}{\eta_{s}}, \frac{\alpha_{I}}{\eta_{I}}\right\}$, and $\alpha=\alpha_{s}$.

Proof. We prove the result for system (2.1). The proof for subsystem (2.2) is similar. Select any $\epsilon>0$. Define the interval $\mathcal{J}=\left[0, \max \left\{S^{0}, \frac{\alpha S^{0}}{A}\right\}+\epsilon\right]$. Apply Lemma A.1 to the $S$ equation of system (2.2), noting that by Lemma 3.1, $\left(x_{s}+x_{I}+\right.$ $y)^{\infty} \leq \frac{\alpha\left(S^{0}-S_{\infty}\right)}{A}$ and $S(t) \in \mathcal{J}$ for all sufficiently large $t$. Since $S_{\infty} \leq S^{\infty} \leq S^{0}$, it follows that

$$
\begin{aligned}
0 & \geq\left(S^{0}-S_{\infty}\right) D-B S_{\infty}\left(x_{s}+x_{I}+y\right)^{\infty} \\
& \geq\left(S^{0}-S_{\infty}\right) D-B S_{\infty}\left(\frac{\alpha\left(S^{0}-S_{\infty}\right)}{A}\right) \\
& \geq\left(S^{0}-S_{\infty}\right)\left(D-\frac{\alpha B S_{\infty}}{A}\right) .
\end{aligned}
$$

Noting the first term is nonnegative, and isolating $S_{\infty}$ on the right hand side, the result follows.

Proof. (of Theorem 3.2). 1. From the $S$ equation of (2.1), either $S(t) \geq S^{0}$ for all sufficiently large $t$ or $S(t)<S^{0}$ for all sufficiently large $t$. In the former case, by Lemma 3.1, $S^{\prime}(t) \leq 0$ for all sufficiently large $t$, and so $S(t) \rightarrow S^{0}$ monotonely, and $x_{s}(t), x_{I}(t), y(t) \rightarrow 0$ as $t \rightarrow \infty$. In the latter case, from the $y$ equation of (2.1), either $y(0)=0$ and the result follows or $y^{\prime}(t)<0$ for all sufficiently large $t$, and hence $y(t)$ converges. By Lemma 3.1 and the $y$ equation, $y: \mathbb{R}_{+} \rightarrow \mathbb{R}$ is a bounded differentiable function and $y^{\prime}$ is uniformly continuously differentiable. By the Barbălat Lemma [2], $\lim _{t \rightarrow \infty} y^{\prime}(t)=0$. If $\lim _{t \rightarrow \infty} y(t)=\bar{\epsilon}>0$, by Lemma 3.1, $S^{\infty}<S^{0} \leq \frac{D_{y}}{\alpha_{y}}$. But then taking the limsup on both sides of the $y$ equation of $(2.1), 0=\bar{\epsilon}\left(-D_{y}+\alpha_{y} S^{\infty}\right)<0$, a contradiction. obtain:

2. Assume that $\frac{D_{y}}{\alpha_{y}}>\frac{D_{I}}{\alpha_{I}}$. Adding the second and third equations of (2.1), we

$$
\left(x_{s}+x_{I}\right)^{\prime}(t) \geq\left(x_{s}+x_{I}\right)(t)\left(-D_{I}+\alpha_{I} S(t)\right)
$$


Integrating both sides of the above inequality with respect to $t$ and rearranging,

$$
\int_{0}^{t} S(t) d t \leq \frac{D_{I} t+\ln \left(\left(x_{s}+x_{I}\right)(t)\right)-\ln \left(\left(x_{s}+x_{I}\right)(0)\right)}{\alpha_{I}} .
$$

Integrating both sides of the $y^{\prime}$ equation of (2.1),

$$
\ln (y(t))=\ln (y(0))-D_{y} t+\alpha_{y} \int_{0}^{t} S(t) d t .
$$

Equations (A.1) and (A.2) imply that

$$
\ln (y(t)) \leq \ln (y(0))+\frac{\alpha_{y}}{\alpha_{I}} \ln \left(\frac{\left(x_{s}+x_{I}\right)(t)}{\left(x_{s}+x_{I}\right)(0)}\right)+\alpha_{y} t\left(\frac{D_{I}}{\alpha_{I}}-\frac{D_{y}}{\alpha_{y}}\right) .
$$

By Lemma 3.1, $y(t), x_{s}(t)$, and $x_{I}(t)$ are non-negative and bounded above for all $t \geq 0$, and so $\liminf _{t \rightarrow \infty} y(t) \geq 0$ and $\lim \sup _{t \rightarrow \infty}\left(x_{s}+x_{I}\right)(t)<\infty$. Therefore, if $\frac{D_{y}}{\alpha_{y}}>\frac{D_{I}}{\alpha_{I}}$, taking the limsup on both sides of (A.3), $\limsup _{t \rightarrow \infty} \ln (y(t))=-\infty$, and so $y(t) \rightarrow 0$ as $t \rightarrow \infty$.

We prove 3 . -5 . for system (2.1). The proof for (2.2) is similar.

3. Assume that $\frac{D_{s}}{\alpha_{s}} \geq S^{0}$. Without loss of generality, assume that $x_{I}(0)>0$ or the result follows from the basic theory of two species competition in the chemostat [38]. Assume also that for all sufficiently large $t, 0 \leq S(t)<S^{0}$. Otherwise, as shown in part 1., $x_{s}(t), x_{I}(t), y(t) \rightarrow 0$ as $t \rightarrow \infty$. Adding the second and third equations of $(2.2)$, given any $\epsilon>0$, for all sufficiently large $t$ :

$$
\begin{aligned}
\left(x_{s}+x_{I}\right)^{\prime}(t) & =x_{s}(t)\left(-D_{s}+\alpha_{s} S(t)\right)+x_{I}(t)\left(-D_{I}+\alpha_{I} S(t)\right) \\
& \leq\left[-D_{s}+\alpha_{s} S(t)\right]\left(x_{s}+x_{I}\right)(t) \\
& \leq\left[-D_{s}+\alpha_{s} \min \left\{S^{0}, S^{\infty}+\epsilon\right\}\right]\left(x_{s}+x_{I}\right)(t) .
\end{aligned}
$$

If $\frac{D_{s}}{\alpha_{s}}>S^{0}$, the term in the square brackets is a negative constant. This implies that $\left(x_{s}+x_{I}\right)(t) \rightarrow 0$ as $t \rightarrow \infty$. Since by Lemma 3.1, $x_{s}(t) \geq 0$ and $x_{I}(t) \geq 0$ for all $t \geq 0$, both $x_{s}(t) \rightarrow 0$ and $x_{I}(t) \rightarrow 0$ as $t \rightarrow \infty$. If $\frac{D_{s}}{\alpha_{S}}=S^{0}$, this implies that the term in the square brackets is nonpositive and so $\lim _{t \rightarrow}\left(x_{s}+x_{I}\right)(t)$ exists. If the limit is zero the result follows as in the previous case. The limit cannot be positive, since then by Lemma 3.1, there exists $\epsilon>0$ such that $S^{\infty}+\epsilon<S^{0}$, and again the term in the square brackets is negative, leading to a contradiction.

4. If $\lim _{t \rightarrow \infty} x_{s}(t)=0$, from the $x_{s}$ equation of (2.1) and Lemma 3.1, $x_{s}$ : $\mathbb{R}_{+} \rightarrow \mathbb{R}$ is a bounded differentiable function and $x_{s}^{\prime}(t)$ is uniformly continuously differentiable. By the Barbălat Lemma $[2], \lim _{t \rightarrow \infty} x_{s}^{\prime}(t)=0$. That $\lim _{t \rightarrow \infty} x_{I}(t)=0$ follows immediately from the $x_{s}$ equation of (2.1).

Integrating the $x_{s}$ and $y$ equations of $(2.1)$, since $\lim _{t \rightarrow \infty} x_{s}(t)=0$, there exists $T>0$ such that $\delta x_{s}(t)<\gamma$ for all $t>T$, and so $x_{s}(t)>x_{s}(T) e^{\int_{T}^{t}-D_{s}+\alpha_{s} S(u) d u}$, and since $\frac{D_{y}}{\alpha_{y}}>\frac{D_{s}}{\alpha_{s}}, y(t)<y(T) e^{\int_{T}^{t}-D_{s}+\alpha_{s} S(u) d u}$. Therefore, using Lemma 3.1, $\lim _{t \rightarrow \infty} y(t)=0$.

5. We argue using proof by contradiction. Assume that $x_{s_{\infty}}=0$, but that $\liminf t \rightarrow \infty x_{I}(t) \neq 0$. Then, $x_{I}^{\infty} \geq x_{I_{\infty}}>0$. Applying Lemma A.1 to the $x_{s}$ equation of (2.1) with $\mathcal{J}$ defined as in the proof of Lemma A.3,

$$
0 \geq x_{s_{\infty}}\left(-D_{s}+\alpha_{s} S_{\infty}-\delta x_{I}^{\infty}\right)+\gamma x_{I_{\infty}}=0+\gamma x_{I_{\infty}}>0
$$


a contradiction. Therefore, $x_{I_{\infty}}=0$.

6. Let $P(t)=\left(S(t), x_{s}(t), x_{I}(t), y(t)\right)$ be any solution of $(2.1)$. It suffices to assume that $\frac{D_{y}}{\alpha_{y}}<S^{0}$ or the result follows by part 1. It also suffices to consider the case that $\limsup _{t \rightarrow \infty} x_{s}(t)>0$, since otherwise the result follows by part 4 . For any such solution, there is a point of the form $\left(S, x_{s}, x_{I}, y\right)$ in the omega limit set of $P(t)$ where $x_{s}>0$ and $x_{I}=0$. Since the closure of the entire orbit though this point must be in the omega limit set of $P(t)$, it follows from the standard results for the chemostat $([38])$ that the equilibrium point $E_{1 x}$ must also be in the omega limit set of $P(t)$, and that it is globally attracting for all points with $x_{s}>0$ and $x_{I}=0$. Using the Butler-McGehee Theorem [28], it follows that if $\lim _{t \rightarrow \infty} x_{I}(t)=0$, this equilibrium point must be the only point in the omega limit set of $P(t)$, and hence $\lim _{t \rightarrow \infty} y(t)=0$.

\section{Appendix B. Proofs: Existence, Uniqueness, and Stability.}

\section{B.1. Existence, Uniqueness, and Local Stability for Subsystem (2.2).}

Existence, Uniqueness, and Local Stability of $E_{0^{*}}$. It is easily seen that $E_{0^{*}}$ always exists and is unique. For the local stability of $E_{0^{*}}$ consider the Jacobian of $(2.2)$ at $E_{0^{*}}$ :

$$
J_{E_{0 *}}=\left[\begin{array}{ccc}
-D & -\frac{\alpha_{s}}{\eta_{s}} S^{0} & -\frac{\alpha_{I}}{\eta_{I}} S^{0} \\
0 & -D_{s}+\alpha_{s} S^{0} & \gamma \\
0 & 0 & -D_{I}+\alpha_{I} S^{0}-\gamma
\end{array}\right]
$$

The eigenvalues are given by the diagonal elements. They are all negative when $S^{0}<\frac{D_{s}}{\alpha_{s}}$ and $S^{0}<\frac{D_{I}+\gamma}{\alpha_{I}}$. Since we assume that $\frac{D_{s}}{\alpha_{s}}<\frac{D_{I}+\gamma}{\alpha_{I}}, E_{0^{*}}$ is locally asymptotically stable if $S^{0}<\frac{D_{s}}{\alpha_{s}}$ and is unstable if $S^{0}>\frac{D_{s}}{\alpha_{s}}$.

Existence, Uniqueness, and Local Stability of $E_{1^{*}} \cdot \bar{S}$ is always positive, and $\bar{x}_{s}$ is positive when $S^{0}>\frac{D_{s}}{\alpha_{s}}$. Hence, $E_{1 *}$ exists when $S^{0}>\frac{D_{s}}{\alpha_{s}}$, and is uniquely defined. To determine the stability of $E_{1^{*}}$, consider the Jacobian matrix of (2.2) evaluated at $E_{1 *}$.

$$
J_{E_{1 *}}=\left[\begin{array}{ccc}
-\frac{\alpha_{s} S^{0} D}{D_{s}} & -\frac{D_{s}}{\eta_{s}} & -\frac{\alpha_{I} D_{s}}{\eta_{I} \alpha_{s}} \\
\alpha_{s}\left(\frac{\eta_{s} S^{0} D}{D_{s}}-\frac{D \eta_{s}}{\alpha_{s}}\right) & 0 & -\delta\left(\frac{\eta_{s} S^{0} D}{D_{s}}-\frac{D \eta_{s}}{\alpha_{s}}\right)+\gamma \\
0 & 0 & -D_{I}+\alpha_{I} \bar{S}+\delta \bar{x}_{s}-\gamma
\end{array}\right] .
$$

To obtain the characteristic equation, evaluate $\operatorname{det}\left(J_{E_{1 *}}-\lambda I\right)$ to obtain:

$$
\left(\lambda+D_{I}-\alpha_{I} \bar{S}-\delta \bar{x}_{s}+\gamma\right)\left(\lambda^{2}+\lambda\left(\frac{\alpha_{s} S^{0} D}{D_{s}}\right)+\alpha_{s} D\left(S^{0}-\frac{D_{s}}{\alpha_{s}}\right)\right)=0
$$

Since both coefficients of the quadratic term are positive, the corresponding eigenvalues have negative real part. The eigenvalue from the linear term is,

$$
\begin{aligned}
-D_{I}+\alpha_{I} \bar{S}+\delta \bar{x}_{s}-\gamma & =-D_{I}+\alpha_{I}\left(\frac{D_{s}}{\alpha_{s}}\right)+\delta\left(\frac{\eta_{s} S^{0} D}{D_{s}}-\frac{D \eta_{s}}{\alpha_{s}}\right)-\gamma \\
& <0 \quad \text { if } \quad S^{0}<\lambda_{c}
\end{aligned}
$$


Therefore, all eigenvalues have negative real parts if $\lambda_{c}>S^{0}$, and at least one eigenvalue with positive real part if $\lambda_{c}<S^{0}$ Hence, when it exists, $E_{1^{*}}$ is locally asymptotically stable when $\lambda_{c}>S^{0}$ and unstable if the inequality is reversed.

Existence, Uniqueness, and Local Stability of $E_{2^{*}}$.

Proof. (of Theorem 3.4). First we consider existence and uniqueness. Substitute the expressions given by (3.2) for $x_{s}^{*}$ and $x_{I}^{*}$ into the $S^{\prime}(t)$ equation of system (2.2) and set $S^{\prime}(t)=0$. The denominator of the resulting expression is always negative for $S<\frac{D_{I}}{\alpha_{I}}$. The numerator of the resulting expression is the cubic:

$$
\begin{aligned}
f(S)= & D \eta_{s} \eta_{I} \delta S^{0} D_{I}+S\left(-S^{0} D \alpha_{I} \eta_{s} \eta_{I} \delta-D \eta_{s} \eta_{I} \delta D_{I}-\alpha_{s} \eta_{I} D_{I} \gamma-\alpha_{s} \eta_{I} D_{I}^{2}\right. \\
& \left.+\alpha_{I} \eta_{s} D_{s} \gamma+\alpha_{I} \eta_{s} D_{s} D_{I}\right)+S^{2}\left(\alpha_{I} D \eta_{s} \eta_{I} \delta+2 \alpha_{s} \alpha_{I} \eta_{I} D_{I}+\alpha_{s} \alpha_{I} \eta_{I} \gamma\right. \\
& \left.-\alpha_{I}^{2} \eta_{s} D_{s}-\alpha_{s} \alpha_{I} \eta_{s} \gamma-\alpha_{s} \alpha_{I} \eta_{s} D_{I}\right)+S^{3} \alpha_{s} \alpha_{I}^{2}\left(\eta_{s}-\eta_{I}\right) .
\end{aligned}
$$

In $\S 3.2$, we proved that $E_{2^{*}}$ exists if and only if there is a root $S^{*}$ of this equation, with $S^{*} \in\left(\frac{D_{s}}{\alpha_{s}}, \frac{D_{I}}{\alpha_{I}}\right)$. It is useful to note that:

$$
\begin{aligned}
f(0) & =D \eta_{s} \eta_{I} \delta S^{0} D_{I}>0 \\
f\left(\frac{D_{s}}{\alpha_{s}}\right) & =D \eta_{s} \eta_{I} \delta\left(D_{I}-\alpha_{I} \frac{D_{s}}{\alpha_{s}}\right)\left(S^{0}-\lambda_{c}\right) \\
f\left(\frac{D_{I}}{\alpha_{I}}\right) & =D_{I} \eta_{s} \gamma\left(D_{s}-\alpha_{s} \frac{D_{I}}{\alpha_{I}}\right)<0
\end{aligned}
$$

We proceed by considering the cases (I) $\eta_{I}=\eta_{s}$, (II) $\eta_{s}>\eta_{I}$, and (III) $\eta_{I}>\eta_{s}$, separately.

(I) First assume that $\eta_{s}=\eta_{I}=\eta$. Then, $f(S)$ reduces to a quadratic equation with $f(0)>0, f\left(\frac{D_{I}}{\alpha_{I}}\right)<0$, and the coefficient of $S^{2}$ is positive. Therefore, both roots are real and positive, and one root lies to the right of $\frac{D_{I}}{\alpha_{I}}$. If $f\left(\frac{D_{s}}{\alpha_{s}}\right)>0$, or equivalently $S^{0}>\lambda_{c}$, as assumed in part 1.(i), there is a unique positive root $S^{*} \in\left(\frac{D_{s}}{\alpha_{s}}, \frac{D_{I}}{\alpha_{I}}\right)$ and hence an equilibrium of the form $E_{2^{*}}$ exists and is uniquely defined. If $S^{0}<\lambda_{c}$, as assumed in part 2.(i), no equilibrium of this form exists.

(II) Next consider $\eta_{s}>\eta_{I}$. The coefficient of $S^{3}$ in the cubic, $f(S)$ is positive. Hence, as $S$ tends to positive (negative) infinity, $f(S)$ tends to positive (negative) infinity. Also, $f(0)>0$, and so there must be at least one negative root of $f(S)$, and $f\left(\frac{D_{I}}{\alpha_{I}}\right)<0$, and so there must be a positive root that is larger than $\frac{D_{I}}{\alpha_{I}}$. This leaves one root, and it lies in $\left(\frac{D_{s}}{\alpha_{s}}, \frac{D_{I}}{\alpha_{I}}\right)$ if and only if $f\left(\frac{D_{s}}{\alpha_{s}}\right)>0$, i.e., if and only if $S^{0}>\lambda_{c}$ as assumed in part 1.(ii). Thus, in this case there is exactly one root in $\left(\frac{D_{s}}{\alpha_{s}}, \frac{D_{I}}{\alpha_{I}}\right)$, and $E_{2^{*}}$ exists and is unique. If $f\left(\frac{D_{s}}{\alpha_{s}}\right) \leq 0$, or equivalently $S^{0} \leq \lambda_{c}$, as assumed in part 2.(i), then no equilibrium of the form $E_{2^{*}}$ exists. (Recall that it is the relative values of $S^{0}$ and $\lambda_{c}$ that determines the local stability of $E_{1^{*}}$, and so we have just shown that if $\eta_{s}>\eta_{I}$, when $E_{2^{*}}$ exists $E_{1^{*}}$ must be unstable.) 
(III) Finally, assume that $\eta_{I}>\eta_{s}$. The condition $S^{0}>\lambda_{c}$ in part 1.(iii), is sufficient to ensure that at least one equilibrium of the form $E_{2^{*}}$ exists, since $f\left(\frac{D_{I}}{\alpha_{I}}\right)<$ 0 and if $S^{0}>\lambda_{c}, f\left(\frac{D_{s}}{\alpha_{s}}\right)>0$. Therefore, there must be at least one root $S^{*} \in$ $\left(\frac{S_{s}}{\alpha)_{s}}, \frac{D_{I}}{\alpha_{I}}\right)$. However up to three distinct values of $S^{*}$ in this interval are possible as illustrated in Figures 3.5-3.6.

Next we show that when $S^{0}>\lambda_{c}$ that $E_{2^{*}}$ is unique when at least one of (3.7) or (3.8) holds. To prove uniqueness when (3.7) holds, we argue as follows. Define $G(S)=\left(S^{0}-S\right) D-\frac{\alpha_{I} x_{I}(S) S}{\eta_{I}}$ and $H(S)=\frac{\alpha_{s} x_{s}(S) S}{\eta_{s}}$, where $x_{s}(S)$ and $x_{I}(S)$ are defined in (3.2). Therefore, $S^{\prime}\left(S^{*}, x_{s}^{*}, x_{I}^{*}\right)=G\left(S^{*}\right)-H\left(S^{*}\right)=0$, and so intersections of $G(S)$ and $H(S)$ for $S \in\left(\frac{D_{s}}{\alpha_{s}}, \frac{D_{I}}{\alpha_{I}}\right)$ are the only candidates for the $S$ component of any equilibrium of the form $E_{2^{*}}$.

$$
\begin{aligned}
\frac{d G}{d S} & =-D-\frac{\alpha_{I} \frac{d x_{I}}{d S} S}{\eta_{I}}-\frac{\alpha_{I} x_{I}}{\eta_{I}} \\
& =-D\left(\frac{\alpha_{I} S}{\eta_{I}}\right)\left(\frac{\gamma \alpha_{I}\left(-D_{s}+\alpha_{s} S\right)+\alpha_{s}\left(-\alpha_{I} S+D_{I}+\gamma\right)\left(D_{I}-\alpha_{I} S\right)}{\delta\left(D_{I}-\alpha_{I} S\right)^{2}}\right) \\
& <0 \quad \text { for } \quad S \in\left(\frac{D_{s}}{\alpha_{s}}, \frac{D_{I}}{\alpha_{I}}\right) \\
\frac{d H}{d S} & =\frac{\alpha_{s} \frac{d x_{s}}{d S} S}{\eta_{s}}+\frac{\alpha_{s} x_{s}}{\eta_{s}} \\
& =\frac{-2 \alpha_{s} \alpha_{I} S+\alpha_{s} D_{I}+\alpha_{s} \gamma}{\delta \eta_{s}} \\
& >0 \text { for } S \in\left(\frac{D_{s}}{\alpha_{s}}, \frac{D_{I}}{\alpha_{I}}\right), \quad \text { when } \quad D_{I}<\gamma .
\end{aligned}
$$

Therefore, $G$ is decreasing for $S \in\left(\frac{D_{s}}{\alpha_{s}}, \frac{D_{I}}{\alpha_{I}}\right)$, and $H$ is increasing there provided that $D_{I}<\gamma$, i.e. (3.7) holds. As well,

$$
\begin{aligned}
& S^{0}>\frac{D_{s}}{D \delta \eta_{s}}\left(D_{I}+\gamma-\frac{\alpha_{I} D_{s}}{\alpha_{s}}+\frac{\delta D \eta_{s}}{\alpha_{s}}\right)=\lambda_{c} \\
\Leftrightarrow & \left(S^{0}-\frac{D_{s}}{\alpha_{s}}\right) D-\frac{\left(D_{I}-\frac{\alpha_{I} D_{s}}{\alpha_{s}}+\gamma\right) D_{s}}{\delta \eta_{s}}>0 \\
\Leftrightarrow & \left(S^{0}-\frac{D_{s}}{\alpha_{s}}\right) D-\frac{\left(D_{I}-\alpha_{I} \frac{D_{s}}{\alpha_{s}}+\gamma\right)\left(-D_{s}+\alpha_{s} \frac{D_{s}}{\alpha_{s}}\right)}{\delta\left(D_{I}-\alpha_{I} \frac{D_{s}}{\alpha_{s}}\right)}\left(\frac{\alpha_{I}}{\eta_{I}} \frac{D_{s}}{\alpha_{s}}\right)>\frac{\left(D_{I}-\alpha_{I} \frac{D_{s}}{\alpha_{s}}+\gamma\right) \alpha_{s} \frac{D_{s}}{\alpha_{s}}}{\delta \eta_{s}} \\
\Leftrightarrow & G\left(\frac{D_{s}}{\alpha_{s}}\right)>H\left(\frac{D_{s}}{\alpha_{s}}\right) .
\end{aligned}
$$

Also, $G\left(\frac{D_{I}}{\alpha_{I}}\right)<\lim _{S \rightarrow \frac{D_{I}^{-}}{\alpha_{I}}} H(S)=\infty$. This ensures one and only one intersection of $G(S)$ and $H(S)$ in $\left(\frac{D_{s}}{\alpha_{s}}, \frac{D_{I}}{\alpha_{I}}\right)$.

To prove uniqueness when (3.8) holds, we use the following argument. Let $g(S)=\frac{\delta D\left(S^{0}-S\right)}{S}$ and $h(S)=\left[\frac{\alpha_{s}}{\eta_{s}}+\frac{\alpha_{I}\left(-D_{s}+\alpha_{s} S\right)}{\eta_{I}\left(D_{I}-\alpha_{I} S\right)}\right]\left(D_{I}+\gamma-\alpha_{I} S\right)$, where $S^{\prime}\left(S^{*}\right)=$ $g\left(S^{*}\right)-h\left(S^{*}\right)=0$. We again find criteria for $g$ and $h$ to have only one intersection for $S \in\left(\frac{D_{s}}{\alpha_{s}}, \frac{D_{I}}{\alpha_{I}}\right)$. 
Clearly $g(S)$ is decreasing.

$$
\begin{aligned}
h^{\prime}(S)= & -\alpha_{I}\left[\frac{\alpha_{s}}{\eta_{s}}+\frac{\alpha_{I}\left(-D_{s}+\alpha_{s} S\right)}{\eta_{I}\left(D_{I}-\alpha_{I} S\right)}\right]+\left(D_{I}+\gamma-\alpha_{I} S\right)\left[\frac{\alpha_{I}\left(\alpha_{s} D_{I}-\alpha_{I} D_{s}\right)}{\eta_{s}\left(D_{I}-\alpha_{I} S\right)^{2}}\right] \\
= & \frac{\alpha_{I}}{\eta_{s} \eta_{I}\left(D_{I}-\alpha_{I} S\right)^{2}}\left[-\alpha_{s} \eta_{I}\left(D_{I}-\alpha_{I} S\right)^{2}+\eta_{s} \alpha_{s}\left(D_{I}-\alpha_{I} S\right)^{2}\right. \\
& \left.+\eta_{s} \gamma\left(\alpha_{s} D_{I}-\alpha_{I} D_{s}\right)\right]
\end{aligned}
$$

The factor on the right in the square bracket is positive when (3.8) holds, and so $h(S)$ is increasing in this case. It remains to show that $g\left(\frac{D_{s}}{\alpha_{s}}\right)>h\left(\frac{D_{s}}{\alpha_{s}}\right)$ and that $g\left(\frac{D_{I}}{\alpha_{I}}\right)<h\left(\frac{D_{I}}{\alpha_{I}}\right)$.

$$
S^{0}>\frac{D_{s}}{D \delta \eta_{s}}\left(D_{I}+\gamma-\frac{\alpha_{I} D_{s}}{\alpha_{s}}+\frac{\delta D \eta_{s}}{\alpha_{s}}\right)=\lambda_{c} \Leftrightarrow g\left(\frac{D_{s}}{\alpha_{s}}\right)>h\left(\frac{D_{s}}{\alpha_{s}}\right) .
$$

Also, $g\left(\frac{D_{I}}{\alpha_{I}}\right)<\lim _{S \rightarrow \frac{D_{I}^{-}}{\alpha_{I}}} h(S)$. This ensures one and only one intersection of $g(S)$ and $h(S)$ in $\left(\frac{D_{s}}{\alpha_{s}}, \frac{D_{I}}{\alpha_{I}}\right)$, when (3.8) holds, i.e., $\eta_{I} \leq \frac{\alpha_{s} \gamma \eta_{s}}{\alpha_{s} D_{I}-\alpha_{I} D_{s}}+\eta_{s}$.

If $S^{0}<\lambda_{c}$, as assumed in part 2.(ii), then $f(0)>0$ and $f\left(\frac{D_{s}}{\alpha_{s}}\right)<0$, and so at least one root must lie in $\left(0, \frac{D_{s}}{\alpha_{s}}\right)$. Thus at most two equilibria of the form $E_{2^{*}}$ are possible, and since $f\left(\frac{D_{I}}{\alpha_{I}}\right)<0$, generically (i.e., unless there is a double root), there is either no equilibrium of this form or there are two.

Next we consider the local stability of $E_{2^{*}}$. Evaluate the Jacobian of $(2.2)$ at $E_{2^{*}}$ to obtain:

$$
J_{E_{2^{*}}}=\left[\begin{array}{ccc}
-\frac{S^{0} D}{S^{*}} & -\frac{\alpha_{s}}{\eta_{s}} S^{*} & -\frac{\alpha_{I}}{\eta_{I}} S^{*} \\
\alpha_{s} x_{s}^{*} & -\gamma \frac{x_{I}^{*}}{x^{*}} & -\delta x_{s}^{*}+\gamma \\
\alpha_{I} x_{I}^{*} & \delta x_{I}^{*} & 0
\end{array}\right],
$$

Evaluate $\operatorname{det}\left(J_{E_{2^{*}}}-\lambda I\right)$ to obtain the characteristic equation. It is of the form $\lambda^{3}+$ $a_{1} \lambda^{2}+a_{2} \lambda+a_{3}=0$, where

$$
\begin{aligned}
& a_{1}=\frac{S^{0} D}{S^{*}}+\frac{\gamma x_{I}^{*}}{x_{s}^{*}}, \quad a_{2}=\frac{\gamma S^{0} D x_{I}^{*}}{x_{s}^{*} S^{*}}+\frac{\alpha_{s}^{2} x_{s}^{*} S^{*}}{\eta_{s}}+\delta x_{I}^{*}\left(\delta x_{s}^{*}-\gamma\right)+\frac{\alpha_{I}^{2} x_{I}^{*} S^{*}}{\eta_{I}}, \\
& a_{3}=\frac{\delta S^{0} D x_{I}^{*}}{S^{*}}\left(\delta x_{s}^{*}-\gamma\right)+\alpha_{s} \alpha_{I} \delta S^{*} x_{s}^{*} x_{I}^{*}\left(\frac{1}{\eta_{I}}-\frac{1}{\eta_{s}}\right) \\
& \quad+\frac{\alpha_{s} \alpha_{I} \gamma S^{*} x_{I}^{*}}{\eta_{s}}+\frac{\alpha_{I}^{2} \gamma S^{*} x_{I}^{* 2}}{\eta_{I} x_{s}^{*}} .
\end{aligned}
$$

By the Routh-Hurwitz Criterion ([16], [25]), all roots of the characteristic equation have negative real part if and only if $a_{1}>0, a_{3}>0$, and $a_{1} a_{2}>a_{3}$. Note that $a_{1}>0$.

Whenever $E_{2^{*}}$ exists, $x_{s}^{*}>\frac{\gamma}{\delta}$. If $\eta_{I} \leq \eta_{s}$, as in part 1.(i),(ii) or 2.(i), the coefficient $a_{3}$ is always positive. When $\eta_{I}>\eta_{s}$ and (3.7) holds

$$
a_{3}=\frac{\delta S^{0} D x_{I}^{*}}{S^{*}}\left(\delta x_{s}^{*}-\gamma\right)+\frac{\alpha_{s} \alpha_{I} \delta S^{*} x_{s}^{*} x_{I}^{*}}{\eta_{I}}-\frac{\alpha_{s} \alpha_{I} \delta S^{*} x_{s}^{*} x_{I}^{*}}{\eta_{s}}+\frac{\alpha_{s} \alpha_{I} \gamma S^{*} x_{I}^{*}}{\eta_{s}}+\frac{\alpha_{I}^{2} \gamma S^{*} x_{I}^{* 2}}{\eta_{I} x_{s}^{*}}
$$




$$
>x_{I}^{*}\left(\delta x_{s}^{*}-\gamma\right)\left(\frac{\delta S^{0} D}{S^{*}}-\frac{\alpha_{I} \alpha_{s} S^{*}}{\eta_{s}}\right) .
$$

Using (3.3) to substitute for $S^{0} D$, in the expression in the brackets, it follows that $a_{3}>0$ if

$$
T=\frac{\delta}{S^{*}}\left(S^{*} D+\frac{\alpha_{s} x_{s}^{*} S^{*}}{\eta_{s}}+\frac{\alpha_{I} x_{I}^{*} S^{*}}{\eta_{I}}\right)-\frac{\alpha_{I} \alpha_{s} S^{*}}{\eta_{s}}>0
$$

Substituting for $x_{s}^{*}$ using (3.2), omitting the terms involving $D$ and $x_{I}^{*}$, and recalling that $S^{*}<\frac{D_{I}}{\alpha_{I}}$, it follows that

$$
\frac{\eta_{s}}{\alpha_{s}} T>\left(D_{I}-\alpha_{I} S^{*}+\gamma\right)-\alpha_{I} S^{*}=D_{I}+\gamma-2 \alpha_{I} S^{*}>D_{I}+\gamma-2 D_{I}=\gamma-D_{I}>0 .
$$

Therefore, if $\eta_{I}>\eta_{s}$ and (3.7) holds, $a_{3}$ is also always positive.

When $\eta_{I}>\eta_{s}$ and (3.8) holds, as assumed in part 3,

$$
a_{3}>\alpha_{I} S^{*} x_{I}^{*}\left(\frac{\alpha_{s} \delta x_{s}^{*}}{\eta_{I}}-\frac{\alpha_{s} \delta x_{s}^{*}}{\eta_{s}}+\frac{\alpha_{s} \gamma}{\eta_{s}}+\frac{\alpha_{I} \gamma x_{I}^{*}}{\eta_{I} x_{s}^{*}}\right) .
$$

Substituting $x_{I}^{*}$ given in (3.2), the expression inside the brackets equals

$$
\left(\frac{1}{\eta_{s} \eta_{I}\left(\delta x_{s}^{*}-\gamma\right)}\right)\left(\left(\delta x_{s}^{*}-\gamma\right)\left(\alpha_{s} \delta \eta_{s} x_{s}^{*}-\alpha_{s} \delta \eta_{I} x_{s}^{*}+\alpha_{s} \gamma \eta_{I}\right)+\alpha_{I} \gamma \eta_{s}\left(-D_{s}+\alpha_{s} S^{*}\right)\right)
$$

Therefore, $a_{3}>0$ if $\alpha_{s} \delta \eta_{s} x_{s}^{*}-\alpha_{s} \delta \eta_{I} x_{s}^{*}+\alpha_{s} \gamma \eta_{I} \geq 0$. Substituting $x_{s}^{*}$ given in (3.2), recalling that $S^{*}>\frac{D_{s}}{\alpha_{s}}$, and rearranging, $a_{3}>0$ when (3.8) holds, since

$$
\begin{aligned}
\alpha_{s} \delta \eta_{s} x_{s}^{*}-\alpha_{s} \delta \eta_{I} x_{s}^{*}+\alpha_{s} \gamma \eta_{I} & =\alpha_{s} \gamma \eta_{I}-\alpha_{s}\left(D_{I}-\alpha_{I} S^{*}+\gamma\right)\left(\eta_{I}-\eta_{s}\right) \\
& \geq \alpha_{s} \gamma \eta_{s}-\left(\alpha_{s} D_{I}-\alpha_{I} D_{s}\right)\left(\eta_{I}-\eta_{s}\right) \\
& \geq 0, \quad \text { when }(3.8) \text { holds. }
\end{aligned}
$$

Next consider the sign of $a_{1} a_{2}-a_{3}$. After using (3.3) to substitute for $S^{0} D$, and then factoring a common denominator, we obtain:

$$
\begin{aligned}
a_{1} a_{2}-a_{3}= & \frac{1}{\eta_{s}^{2} \eta_{I}^{2} x_{s}^{* 2}}\left(\eta_{s}^{2} \eta_{I}^{2} \gamma^{2} x_{I}^{* 2} D+\eta_{s} \eta_{I}^{2} \gamma^{2} \alpha_{s} x_{s}^{*} x_{I}^{* 2}+\eta_{s}^{2} \eta_{I} \gamma^{2} \alpha_{I} x_{I}^{* 3}\right. \\
& +D^{2} \eta_{s}^{2} \eta_{I}^{2} \gamma x_{s}^{*} x_{I}^{*}+2 \eta_{s} \eta_{I}^{2} \gamma \alpha_{s} x_{s}^{* 2} x_{I}^{*} D+2 \eta_{s}^{2} \eta_{I} \gamma \alpha_{I} x_{s}^{*} x_{I}^{* 2} D \\
& +\eta_{I}^{2} \gamma \alpha_{s}^{2} x_{s}^{* 3} x_{I}^{*}+2 \eta_{s} \eta_{I} \gamma \alpha_{s} \alpha_{I} x_{s}^{* 2} x_{I}^{* 2}+\eta_{s}^{2} \gamma \alpha_{I}^{2} x_{s}^{*} x_{I}^{* 3} \\
& +\eta_{s} \eta_{I}^{2} \alpha_{s}^{2} x_{s}^{* 3} S^{*} D+\eta_{I}^{2} \alpha_{s}^{3} x_{s}^{* 4} S^{*}+\eta_{s}^{2} \eta_{I} \alpha_{I}^{2} x_{s}^{* 2} x_{I}^{*} S^{*} D \\
& +\eta_{s} \eta_{I} \alpha_{s} \alpha_{I} x_{s}^{* 3} x_{I}^{*}\left(\alpha_{s}+\alpha_{I}-\eta_{s} \delta+\eta_{I} \delta\right)+\eta_{s}^{2} \alpha_{I}^{3} x_{s}^{* 2} x_{I}^{* 2} S^{*} \\
& \left.+\eta_{s}^{2} \eta_{I}^{2} \gamma \delta x_{s}^{*} x_{I}^{* 2}\left(\delta x_{s}^{*}-\gamma\right)+\eta_{s} \eta_{I}^{2} \gamma x_{s}^{* 2} x_{I}^{*}\left(\alpha_{s}-\alpha_{I}\right)\right) .
\end{aligned}
$$

Since $\alpha_{s} \geq \alpha_{I}$, and $x_{s}^{*}>\frac{\gamma}{\delta}$ whenever $E_{2 *}$ exists, a sufficient condition for $a_{1} a_{2}-$ $a_{3}>0$ is that $\frac{\alpha_{I}+\alpha_{s}}{\delta} \geq \eta_{s}-\eta_{I}$. Note that this always holds when $\eta_{I} \geq \eta_{s}$ as assumed in part 1.(i),(iii) or 2.(ii).

\section{$(2.2)$.}

B.2. Proofs: Criteria in Table 3.1 - Global Stability for Subsystem 
Global stability of $E_{0^{*}}$. The global stability of $E_{0^{*}}$ when $\frac{D_{s}}{\alpha_{S}}>S^{0}$ follows immediately from Theorem 3.2 parts 3 . and 4 .

REMARK B.1. Note that $E_{0^{*}}$ is globally asymptotically stable whenever it is locally asymptotically stable. Otherwise, for all sufficiently large $t$, for all solutions of (2.2), $0 \leq S(t) \leq S^{0}$.

Global stability of $E_{1^{*}}$. Consider the following Lyapunov function:

$$
V\left(S, x_{s}, x_{I}\right)=S-\bar{S}-\bar{S}(\ln (S)-\ln (\bar{S}))+k_{1}\left[x_{s}-\bar{x}_{s}-\bar{x}_{s}\left(\ln \left(x_{s}\right)-\ln \left(\bar{x}_{s}\right)\right)\right]+k_{2} x_{I}
$$

with $k_{1}, k_{2}>0$ positive constants to be determined.

$$
\begin{aligned}
\dot{V}= & \left(\frac{S-\bar{S}}{S}\right) \dot{S}+k_{1} \dot{x_{s}}\left(\frac{x_{s}-\bar{x}_{s}}{x_{s}}\right)+k_{2} \dot{x_{I}} \\
= & \left(\frac{S-\bar{S}}{S}\right)\left[\left(S^{0}-S\right) D-\frac{\alpha_{s} x_{s} S}{\eta_{s}}-\frac{\alpha_{I} x_{I} S}{\eta_{I}}\right] \\
& +k_{1}\left(\frac{x_{s}-\bar{x}_{s}}{x_{s}}\right)\left[x_{s}\left(-D_{s}+\alpha_{s} S\right)-\delta x_{s} x_{I}\right]+k_{1} \gamma x_{I}\left(\frac{x_{s}-\bar{x}_{s}}{x_{s}}\right) \\
& +k_{2}\left[x_{I}\left(-D_{I}+\alpha_{I} S\right)+\delta x_{s} x_{I}-\gamma x_{I}\right] \\
= & \left(\frac{S-\bar{S}}{S}\right)\left[\left(S^{0}-S\right) D-\frac{\alpha_{s} \overline{x_{s}} S}{\eta_{s}}\right] \\
& -\alpha_{s}\left(\frac{1}{\eta_{s}}\right)(S-\bar{S})\left(x_{s}-\bar{x}_{s}\right)+k_{1} \alpha_{s}\left(x_{s}-\bar{x}_{s}\right)(S-\bar{S}) \\
& +k_{2} x_{I}\left[-D_{I}+\alpha_{I} S+\delta x_{s}-\gamma+\gamma \frac{k_{1}}{k_{2}}-\gamma \frac{k_{1}}{k_{2}}\left(\frac{\bar{x}_{s}}{x_{s}}\right)-\frac{k_{1}}{k_{2}} \delta x_{s}+\frac{k_{1}}{k_{2}} \delta \bar{x}_{s}-\frac{S \alpha_{I}}{k_{2} \eta_{I}}+\frac{\bar{S} \alpha_{I}}{k_{2} \eta_{I}}\right] \\
= & T 1+\alpha_{s}(S-\bar{S})\left(x_{s}-\bar{x}_{s}\right)\left(k_{1}-\frac{1}{\eta_{s}}\right)+k_{2} x_{I}\left(-D_{I}+\frac{\alpha_{I} \bar{S}}{k_{2} \eta_{I}}+\frac{k_{1}}{k_{2}} \delta \bar{x}_{s}-\gamma+\gamma \frac{k_{1}}{k_{2}}\right) \\
& +k_{2} x_{I}\left[\alpha_{I} S\left(1-\frac{1}{k_{2} \eta_{I}}\right)+\delta x_{s}\left(1-\frac{k_{1}}{k_{2}}\right)-\gamma \frac{k_{1}}{k_{2}}\left(\frac{\bar{x}_{s}}{x_{s}}\right)\right]
\end{aligned}
$$

where $T 1=\left(\frac{S-\bar{S}}{S}\right)\left[\left(S^{0}-S\right) D-\frac{\alpha_{s} \overline{x_{s} S}}{\eta_{s}}\right] \leq 0$, with equality if and only if $S=\bar{S}$. Let $k_{1}=\frac{1}{\eta_{s}}$, to eliminate the second term, and $k_{2}=\frac{1}{\eta_{s}}$. Then,

$$
\begin{aligned}
\dot{V}= & T 1+\frac{1}{\eta_{s}} x_{I}\left(-D_{I}+\frac{\eta_{s}}{\eta_{I}}\left(\alpha_{I} \bar{S}\right)+\delta \bar{x}_{s}\right) \\
& +\frac{1}{\eta_{s}} x_{I}\left[\alpha_{I} S\left(1-\frac{\eta_{s}}{\eta_{I}}\right)-\gamma\left(\frac{\bar{x}_{s}}{x_{s}}\right)\right] .
\end{aligned}
$$

The third term is always non-positive. If $D_{I}$ is sufficiently large, then the second term is non-positive, and $\dot{V} \leq 0$ with equality if and only if $S=\bar{S}$ and $x_{I}=0$. Since by Lemma 3.1 all solutions are bounded, by the LaSalle Extension Theorem, every solution of (2.2) approaches $\mathcal{M}$, the largest invariant subset of

$$
\Omega=\left\{\left(S, x_{s}, x_{I}\right) \in \mathbb{R}_{+}^{3}: \dot{V}\left(S, x_{s}, x_{I}\right)=0\right\}=\left\{\left(S, x_{s}, x_{I}\right) \in \mathbb{R}_{+}^{3}: S=\bar{S}, x_{I}=0\right\} .
$$

To be in $\mathcal{M}$, since $S=\bar{S}$, must be constant, $S^{\prime}(t) \equiv 0$ must hold, and since $x_{I}=0$, it follows that $x_{s}(t) \equiv \bar{x}_{s}$. Hence, $\mathcal{M}=\left(\bar{S}, \bar{x}_{s}, 0\right)=E_{1^{*}}$, and so $E_{1^{*}}$ is globally asymptotically stable when it is locally asymptotically stable provided that in addition, $D_{I}$ is sufficiently large. 
REMARK B.2. If $\eta_{s}<\eta_{I}$, then take $k_{2}=\frac{1}{\eta_{I}}$.

$$
\begin{aligned}
\dot{V}= & T 1+\frac{1}{\eta_{I}} x_{I}\left(-D_{I}+\alpha_{I} \bar{S}+\frac{\eta_{I}}{\eta_{s}}\left(\delta \bar{x}_{s}\right)-\gamma\left(1-\frac{\eta_{I}}{\eta_{s}}\right)\right) \\
& +\frac{1}{\eta_{I}} x_{I}\left[\delta x_{s}\left(1-\frac{\eta_{I}}{\eta_{s}}\right)-\frac{\eta_{I} \gamma}{\eta_{s}}\left(\frac{\bar{x}_{s}}{x_{s}}\right)\right]
\end{aligned}
$$

A similar argument applies. Again $E_{1 *}$ is globally asymptotically stable when it is locally asymptotically stable provided that in addition, $D_{I}$ is sufficiently large.

Global stability of $E_{2^{*}}$. In the special case that $\eta_{s}=\eta_{I}=\eta$ and $D=D_{s}=D_{I}$, if $z(t)=S^{0}-S(t)-\frac{x_{s}(t)}{\eta}-\frac{x_{I}(t)}{\eta}$, then

$$
z^{\prime}(t)=-S^{0} D+S(t) D+\frac{x_{s}(t) D}{\eta}+\frac{x_{I}(t) D}{\eta}=-D z(t) .
$$

This implies that $z(t)=z(0) e^{-D t}$, and so as $t \rightarrow \infty, z(t) \rightarrow 0$ and hence $S(t)+\frac{x_{s}(t)}{\eta}+$ $\frac{x_{I}(t)}{\eta} \rightarrow S^{0}$. Therefore we can consider the two dimensional limiting system obtained from (2.2) by replacing $S(t)$ by $S(t)=S^{0}-\frac{x_{s}(t)}{\eta}-\frac{x_{I}(t)}{\eta}$ :

$$
\begin{aligned}
& x_{s}^{\prime}(t)=x_{s}(t)\left(-D+\alpha_{s}\left(S^{0}-\frac{x_{s}(t)}{\eta}-\frac{x_{I}(t)}{\eta}\right)\right)-\delta x_{s}(t) x_{I}(t)+\gamma x_{I}(t) \equiv f_{1}\left(x_{s}, x_{I}\right) \\
& x_{I}^{\prime}(t)=x_{I}(t)\left(-D+\alpha_{I}\left(S^{0}-\frac{x_{s}(t)}{\eta}-\frac{x_{I}(t)}{\eta}\right)\right)+\delta x_{s}(t) x_{I}(t)-\gamma x_{I}(t) \equiv f_{2}\left(x_{s}, x_{I}\right) \\
& \left(\text { B.8) for }\left\{\left(x_{s}, x_{I}\right): x_{s} \geq 0, x_{I} \geq 0, \frac{x_{s}}{\eta}+\frac{x_{I}}{\eta} \leq S^{0}\right\} .\right.
\end{aligned}
$$

System (B.8) has three equilibria, $X_{1}=(0,0), X_{2}=\left(\frac{\eta}{\alpha_{s}}\left(-D+\alpha_{s} S^{0}\right), 0\right)$, and $X_{3}=$ $\left(x_{s}^{*}, x_{I}^{*}\right)$, with $x_{s}^{*}$ and $x_{I}^{*}$ defined in (3.2). The local stability results of subsystem (2.2) apply to (B.8), and so when $\frac{D}{\alpha_{s}}<S^{*}<\frac{D}{\alpha_{I}}$ and $\frac{\gamma}{\delta}<x_{s}^{*}<\frac{D+\gamma}{\delta}, X_{1}$ is a repeller, $X_{2}$ is a saddle point, with stable manifold restricted to the $x_{s}$-axis, and $X_{3}$ is locally asymptotically stable. Applying the Dulac criterion with auxiliary function $\beta\left(x_{s}, x_{I}\right)=\frac{1}{x_{s} x_{I}}$ on $\left\{\left(x_{s}, x_{I}\right): x_{s}>0, x_{I}>0\right\}$,

$$
\frac{\partial}{\partial x_{s}}\left(\beta f_{1}\right)+\frac{\partial}{\partial x_{I}}\left(\beta f_{2}\right)=-\frac{\alpha_{s}}{x_{I} \eta}-\frac{\gamma}{x_{s}^{2}}-\frac{\alpha_{I}}{x_{s} \eta}<0 .
$$

It follows that (B.8) has no nontrivial periodic orbits. By Lemma 3.1, all solutions are bounded, and so by the Poincaré-Bendixson Theorem, $X_{3}$ is a globally asymptotically stable with respect to solutions with $x_{s}(0)>0, x_{I}(0)>0$, and $\frac{1}{\eta}\left(x_{s}(0)+x_{I}(0)\right)<S^{0}$. There are no cycles of rest points, since the only trajectories connecting equilibria go from $X_{1}$ to $X_{2}$ to $X_{3}$, and cannot leave $X_{3}$, since $X_{3}$ is locally asymptotically stable. Since all five hypotheses (H1) - (H5) are of Theorem F.1 of [28] are satisfied, $E_{2^{*}}$ is globally asymptotically stable for subsystem (2.2).

\section{B.3. Existence, Uniqueness, and Local Stability for System (2.1).}

Existence, Uniqueness, and Local Stability of $E_{0}$. $E_{0}$ always exists. The Jacobian of (2.1) evaluated at $E_{0}$ is:

$$
J_{E_{0}}=\left[\begin{array}{cccc}
-D & -\frac{\alpha_{s}}{\eta_{s}} S^{0} & -\frac{\alpha_{I}}{\eta_{I}} S^{0} & \frac{\alpha_{y}}{\eta_{y}} S^{0} \\
0 & -D_{s}+\alpha_{s} S^{0} & \gamma & 0 \\
0 & 0 & -D_{I}+\alpha_{I} S^{0}-\gamma & 0 \\
0 & 0 & 0 & -D_{y}+\alpha_{y} S^{0}
\end{array}\right]
$$


The eigenvalues of $J_{E_{0}}$ are on the diagonal. $E_{0}$ is locally asymptotically stable when $S^{0}<\min \left(\frac{D_{s}}{\alpha_{s}}, \frac{D_{y}}{\alpha_{y}}\right)$, and is unstable if $S^{0}>\frac{D_{y}}{\alpha_{y}}$ or $S^{0}>\frac{D_{s}}{\alpha_{s}}$.

Existence, Uniqueness, and Local Stability of $E_{1 x}$. Just as for $E_{1^{*}}, E_{1 x}$ exists when $\frac{D_{s}}{\alpha_{s}}<S^{0}$. For the local stability the Jacobian of (2.1) evaluated at $E_{1 x}$ is:

$$
J_{E_{1 x}}=\left[\begin{array}{cccc} 
& & & -\frac{\alpha_{y} D_{s}}{\eta_{y} \alpha_{s}} \\
& J_{E_{1 *}} & 0 \\
0 & 0 & 0 & -D_{y}+\alpha_{y}\left(\frac{D_{s}}{\alpha_{s}}\right)
\end{array}\right] .
$$

The eigenvalues of this matrix are the eigenvalues of the $3 \times 3$ sub-matrix $E_{1^{*}}$ and $-D_{y}+\alpha_{y}\left(\frac{D_{s}}{\alpha_{s}}\right)$. This eigenvalue is negative if $\frac{D_{s}}{\alpha_{s}}<\frac{D_{y}}{\alpha_{y}}$, and positive if the inequality is reversed and so asymptotically stable when $\frac{D_{s}}{\alpha_{s}}<\frac{D_{y}}{\alpha_{y}}$ and $E_{1^{*}}$ is asymptotically stable, i.e., $S^{0}<\lambda_{c}$.

Existence, Uniqueness, and Local Stability of $E_{1 y}$. $E_{1 y}$ exists when $S^{0}>\frac{D_{y}}{\alpha_{y}}$. The Jacobian of (2.1) at $E_{1 y}$ is:

$$
J_{E_{1 y}}=\left[\begin{array}{cccc}
-\frac{\alpha_{y} S^{0} D}{D_{y}} & -\frac{\alpha_{s} D_{y}}{\eta_{s} \alpha_{y}} & -\frac{\alpha_{I} D_{y}}{\eta_{I} \alpha_{y}} & -\frac{D_{y}}{\eta_{y}} \\
0 & -D_{s}+\frac{\alpha_{s} D_{y}}{\alpha_{y}} & \gamma & 0 \\
0 & 0 & -D_{I}+\alpha_{I}\left(\frac{D_{y}}{\alpha_{y}}\right)-\gamma & 0 \\
\left(\frac{\alpha_{y} D \eta_{y}}{D_{y}}\right)\left(S^{0}-\frac{D_{y}}{\alpha_{y}}\right) & 0 & 0 & 0
\end{array}\right] .
$$

To find the characteristic equation, evaluate $\operatorname{det}\left(J_{E_{1 y}}-\lambda I\right)$ to obtain:

$$
\left(D_{I}-\frac{\alpha_{I} D_{y}}{\alpha_{y}}+\gamma+\lambda\right)\left(D_{s}-\frac{\alpha_{s} D_{y}}{\alpha_{y}}+\lambda\right)\left[\lambda^{2}+\frac{\alpha_{y} S^{0} D}{D_{y}} \lambda+\alpha_{y} D\left(S^{0}-\frac{D_{y}}{\alpha_{y}}\right)\right]=0
$$

Two eigenvalues $\alpha_{I}\left(\frac{D_{y}}{\alpha_{y}}-\frac{D_{I}+\gamma}{\alpha_{I}}\right)$ and $\alpha_{s}\left(\frac{D_{y}}{\alpha_{y}}-\frac{D_{s}}{\alpha_{s}}\right)$ are given by the linear terms. Since we are assuming throughout that $\frac{D_{s}}{\alpha_{s}}<\frac{D_{I}}{\alpha_{I}}$, they are both negative when $\frac{D_{y}}{\alpha_{y}}<\frac{D_{s}}{\alpha_{s}}$. Both roots of the quadratic term have negative real part by the RouthHurwitz Criterion, since the coefficient $\frac{\alpha_{y} D S^{0}}{D_{y}}$, is always positive; and the constant term $\alpha_{y} D\left(S^{0}-\frac{D_{y}}{\alpha_{y}}\right)$, is positive whenever $E_{1 y}$ exists. Hence, $E_{1 y}$ is locally asymptotically stable when it exists and $\frac{D_{y}}{\alpha_{y}}<\frac{D_{s}}{\alpha_{s}}$ and unstable if $\frac{D_{y}}{\alpha_{y}}>\frac{D_{s}}{\alpha_{s}}$.

Existence, Uniqueness, and Local Stability of $E_{2}$. The first three components of $E_{2}$ are identical to those of $E_{2^{*}}$ and so the number of equilibria that exist of the form $E_{2}$ is identical to the number of equilibria that exist of the form $E_{2 *}$.

For the local stability of $E_{2}$, evaluate the Jacobian of (2.1) at $E_{2}$ to obtain:

$$
J_{E_{2}}=\left[\begin{array}{cccc} 
& & & -\frac{\alpha_{y}}{\eta_{y}} S^{*} \\
& J_{E_{2^{*}}} & 0 \\
0 & 0 & 0 & -D_{y}+\alpha_{y} S^{*}
\end{array}\right] .
$$


Therefore, three of the characteristic roots are the same as those for $E_{2^{*}}$, and the fourth root is $-D_{y}+\alpha_{y} S^{*}$. Therefore, $E_{2}$ is locally asymptotically stable, if $S^{*}<\frac{D_{y}}{\alpha_{y}}$ and the corresponding equilibrium of the form $E_{2^{*}}$ is locally asymptotically stable.

Existence, Uniqueness, and Local Stability of $E_{3}$. It is clear that only one equilibrium of the form $E_{3}$ can exist, since its components are each uniquely defined in (3.4). $\widehat{S}=\frac{D_{y}}{\alpha_{y}}$ is always positive; $\widehat{x}_{s}$ is positive when $\frac{D_{y}}{\alpha_{y}}<\frac{D_{I}+\gamma}{\alpha_{I}} ; \widehat{x}_{I}$ is positive when $\frac{D_{s}}{\alpha_{s}}<\frac{D_{y}}{\alpha_{y}}<\frac{D_{I}}{\alpha_{I}}$; and $\widehat{y}$ is positive when

$$
S^{0}>\frac{D_{y}}{\alpha_{y} D}\left[\frac{\alpha_{s}\left(D_{I}+\gamma-\frac{\alpha_{I} D_{y}}{\alpha_{y}}\right)}{\delta \eta_{s}}+\frac{\alpha_{I}\left(D_{I}+\gamma-\frac{\alpha_{I} D_{y}}{\alpha_{y}}\right)\left(-D_{s}+\frac{\alpha_{s} D_{y}}{\alpha_{y}}\right)}{\delta \eta_{I}\left(D_{I}-\frac{\alpha_{I} D_{y}}{\alpha_{y}}\right)}+D\right] \text {. }
$$

Hence, $E_{3}$ exists when $\frac{D_{s}}{\alpha_{s}}<\frac{D_{y}}{\alpha_{y}}<\frac{D_{I}}{\alpha_{I}}$, and

$$
S^{0}>\frac{D_{y}}{\alpha_{y} D}\left(D+\frac{\alpha_{s}}{\delta \eta_{I} \eta_{s}}\left(D_{I}+\gamma-\frac{\alpha_{I} D_{y}}{\alpha_{y}}\right)\left(\eta_{I}+\eta_{s} \frac{\left(\frac{D_{y}}{\alpha_{y}}-\frac{D_{s}}{\alpha_{s}}\right)}{\left(\frac{D_{I}}{\alpha_{I}}-\frac{D_{y}}{\alpha_{y}}\right)}\right)\right) .
$$

Next consider the local stability of $E_{3}$ in the special case: $\eta_{s}=\eta_{I}=\eta$ and $D=D_{s}=D_{I}=D_{y}$. Restricting the parameters thus, simplifies the analysis. Let $z(t)=S^{0}-S(t)-\frac{x_{s}(t)}{\eta}-\frac{x_{I}(t)}{\eta}-\frac{y(t)}{\eta_{y}}$. Then

$$
z^{\prime}(t)=-S^{0} D+S(t) D+\frac{x_{s}(t) D}{\eta}+\frac{x_{I}(t) D}{\eta}+\frac{y(t) D}{\eta_{y}}=-D z(t) .
$$

This implies that $z(t)=z(0) e^{-D t}$, and so as $t \rightarrow \infty, z(t) \rightarrow 0$ and hence $S(t)+\frac{x_{s}(t)}{\eta}+$ $\frac{x_{I}(t)}{\eta}+\frac{y(t)}{\eta_{y}} \rightarrow S^{0}$. Therefore we can consider the three dimensional limiting system obtained from (2.1) by replacing $S(t)$ by $S(t)=S^{0}-\frac{x_{s}(t)}{\eta}-\frac{x_{I}(t)}{\eta}-\frac{y(t)}{\eta_{y}}$ :

$$
\begin{aligned}
x_{s}^{\prime}(t)= & x_{s}(t)\left(-D+\alpha_{s}\left(S^{0}-\frac{x_{s}(t)}{\eta}-\frac{x_{I}(t)}{\eta}-\frac{y(t)}{\eta_{y}}\right)\right) \\
& -\delta x_{s}(t) x_{I}(t)+\gamma x_{I}(t) \\
x_{I}^{\prime}(t)= & x_{I}(t)\left(-D+\alpha_{I}\left(S^{0}-\frac{x_{s}(t)}{\eta}-\frac{x_{I}(t)}{\eta}-\frac{y(t)}{\eta_{y}}\right)\right) \\
& +\delta x_{s}(t) x_{I}(t)-\gamma x_{I}(t) \\
y^{\prime}(t)= & y(t)\left(-D+\alpha_{y}\left(S^{0}-S(t)-\frac{x_{s}(t)}{\eta}-\frac{x_{I}(t)}{\eta}-\frac{y(t)}{\eta_{y}}\right)\right)
\end{aligned}
$$

This system has the corresponding interior equilibrium $E_{3}=\left(\widehat{x}_{s}, \widehat{x}_{I}, \widehat{y}\right)$. The three eigenvalues of this system have the same sign as three eigenvalues of the full four dimensional system; the fourth eigenvalue is negative. The Jacobian matrix for (B.9) evaluated at $E_{3}$ is:

$$
J_{E_{3}}=\left[\begin{array}{ccc}
-\frac{\alpha_{s} \widehat{x}_{s}}{\eta}-\frac{\gamma \widehat{x}_{I}}{\widehat{x}_{s}} & -\frac{\alpha_{s} \widehat{x}_{s}}{\eta}-\delta \widehat{x}_{s}+\gamma & -\frac{\alpha_{s} \widehat{x}_{s}}{\eta_{y}} \\
\frac{\alpha_{I} \widehat{x}_{I}}{\eta}+\delta \widehat{x}_{I} & -\frac{\alpha_{I} \widehat{x}_{I}}{\eta} & -\frac{\alpha_{I} \widehat{x}_{I}}{\eta_{y}} \\
-\frac{\alpha_{y} \widehat{y}}{\eta} & -\frac{\alpha_{y} \widehat{y}}{\eta} & -\frac{\alpha_{y} \widehat{y}}{\eta_{y}}
\end{array}\right] .
$$


The characteristic equation is of the form $\lambda^{3}+a_{1} \lambda^{2}+a_{2} \lambda+a_{3}=0$, where

$$
\begin{aligned}
& a_{1}=\frac{\alpha_{s} \widehat{x}_{s}}{\eta}+\frac{\gamma \widehat{x}_{I}}{\widehat{x}_{s}}+\frac{\alpha_{I} \widehat{x}_{I}}{\eta}+\frac{\alpha_{y} \widehat{y}}{\eta_{y}}, \\
& a_{2}=\frac{\gamma \widehat{x}_{I}}{\widehat{x}_{s}}\left(\frac{\alpha_{I} \widehat{x}_{I}}{\eta}+\frac{\alpha_{y} \widehat{y}}{\eta_{y}}\right)+\frac{\delta \widehat{x}_{s} \widehat{x}_{I}}{\eta}\left(\alpha_{s}-\alpha_{I}\right)+\delta \widehat{x}_{I}\left(\delta \widehat{x}_{s}-\gamma\right)+\frac{\gamma \alpha_{I} \widehat{x}_{I}}{\eta}, \quad \text { and } \\
& a_{3}=\frac{\delta \alpha_{y} \widehat{x}_{I} \widehat{y}}{\eta_{y}}\left(\delta \widehat{x}_{s}-\gamma\right) .
\end{aligned}
$$

Clearly, $a_{1}>0$. Since $\alpha_{s} \geq \alpha_{I}$, when $E_{3}$ exists, i.e. $\widehat{x}_{s}>\frac{\gamma}{\delta}$, then $a_{3}>0$. Now,

$$
\begin{aligned}
a_{1} a_{2}-a_{3}= & \left(\frac{\alpha_{s} \widehat{x}_{s}}{\eta}+\frac{\gamma \widehat{x}_{I}}{\widehat{x}_{s}}+\frac{\alpha_{I} \widehat{x}_{I}}{\eta}\right)\left[\frac{\gamma \widehat{x}_{I}}{\widehat{x}_{s}}\left(\frac{\alpha_{I} \widehat{x}_{I}}{\eta}+\frac{\alpha_{y} \widehat{y}}{\eta_{y}}\right)+\frac{\delta \widehat{x}_{s} \widehat{x}_{I}}{\eta}\left(\alpha_{s}-\alpha_{I}\right)+\frac{\gamma \alpha_{I} \widehat{x}_{I}}{\eta}\right. \\
& \left.+\delta \widehat{x}_{I}\left(\delta \widehat{x}_{s}-\gamma\right)\right]+\frac{\alpha_{y} \widehat{y}}{\eta_{y}}\left[\frac{\gamma \widehat{x}_{I}}{\widehat{x}_{s}}\left(\frac{\alpha_{I} \widehat{x}_{I}}{\eta}+\frac{\alpha_{y} \widehat{y}}{\eta_{y}}\right)+\frac{\delta \widehat{x}_{s} \widehat{x}_{I}}{\eta}\left(\alpha_{s}-\alpha_{I}\right)+\frac{\gamma \alpha_{I} \widehat{x}_{I}}{\eta}\right]
\end{aligned}
$$

$>0$.

Hence, under these restrictions on the parameters, by the Routh-Hurwitz criterion, $E_{3}$ is locally asymptotically stable whenever it exists.

Proof. (of Theorem 3.5.) 1. If an equilibrium of the form $E_{3}$ exists, $S^{0}>\frac{D_{y}}{\alpha_{y}}>\frac{D_{s}}{\alpha_{s}}$ (see Table 3.1), and hence $E_{1 x}$ and $E_{1 y}$ exist. It remains to show that if an equilibrium of the form $E_{3}$ exists, at least one equilibrium of the form $E_{2}$ also exists.

Any equilibrium of the form $E_{2}$ or $E_{3}$ must have components satisfying $x_{s}(S)=$ $\frac{-\alpha_{I} S+D_{I}+\gamma}{\delta}>0, x_{I}(S)=\frac{x_{S}\left(-D_{s}+\alpha_{s} S\right)}{\delta x_{s}-\gamma}>0$ and $y(S)=\frac{\eta_{y}}{\alpha_{y}}\left(\frac{S^{0} D}{S}-D-\frac{\alpha_{s} x_{s}}{\eta_{s}}-\frac{\alpha_{I} x_{I}}{\eta_{I}}\right)$, where $y(\widehat{S})>0$ for $E_{3}$ and $y\left(S^{*}\right)=0$ for $E_{2}$.

Define a function

$$
H(S)=\left(S^{0}-S\right) D-\frac{\alpha_{s} S x_{s}(S)}{\eta_{s}}-\frac{\alpha_{I} S x_{I}(S)}{\eta_{I}} .
$$

Then $H(\widehat{S})>0$, since

$$
\left(S^{0}-\widehat{S}\right) D-\frac{\alpha_{s} \widehat{S} x_{s}(\widehat{S})}{\eta_{s}}-\frac{\alpha_{I} \widehat{S} x_{I}(\widehat{S})}{\eta_{I}}-\frac{\alpha_{y} \widehat{S} y(\widehat{S})}{\eta_{y}}=0
$$

and $y(\widehat{S})>0$.

For $S \in\left(\widehat{S}, \frac{D_{I}}{\alpha_{I}}\right), H(S)$ is a continuous function of $S$. Note that $x_{s}\left(\frac{D_{I}}{\alpha_{I}}\right)>0$ and $\lim _{S \rightarrow{\frac{D_{I}}{\alpha_{I}}}^{-}} x_{I}(S)=+\infty$, and so $\lim _{S \rightarrow{\frac{D_{I}}{\alpha_{I}}}^{-}} H(S)=-\infty$, and that if $\frac{D_{s}}{\alpha_{s}}<S^{0}<\frac{D_{I}}{\alpha_{I}}$, then since $x_{s}(S)>0$ and $x_{I}(S)>0$ for $S \in\left[\widehat{S}, S^{0}\right]$, it follows that $H\left(S^{0}\right)<0$. Hence, there exists a value of $S^{*} \in\left(\widehat{S}, \min \left(S^{0}, \frac{D_{I}}{\alpha_{I}}\right)\right)$ satisfying $H\left(S^{*}\right)=0, x_{s}\left(S^{*}\right)>0$, and $x_{I}\left(S^{*}\right)>0$. Therefore, an equilibrium of the form $E_{2}$ exists.

2. By part 1., we can assume that if $E_{3}$ exists an equilibrium of the form $E_{2}$ also exists. Differentiating the expressions for the components $x_{s}(S), x_{I}(S)$, and $y(S)$ 
given in part 1 ., with respect to $S$,

$$
\begin{aligned}
\frac{d x_{s}}{d S}= & \frac{-\alpha_{I}}{\delta}, \\
\frac{d x_{I}}{d S}= & \frac{\left[\left(\frac{-\alpha_{I}}{\delta}\right)\left(-D_{s}+\alpha_{s} S\right)+\alpha_{s} x_{s}\right]\left(\delta x_{s}-\gamma\right)+\delta\left(\frac{\alpha_{I}}{\delta}\right) x_{s}\left(-D_{s}+\alpha_{s} S\right)}{\left(\delta x_{s}-\gamma\right)^{2}} \\
= & \frac{\gamma \alpha_{I}\left(-D_{s}+\alpha_{s} S\right)+\alpha_{s}\left(-\alpha_{I} S+D_{I}+\gamma\right)\left(D_{I}-\alpha_{I} S\right)}{\delta\left(D_{I}-\alpha_{I} S\right)^{2}}, \\
\frac{d y}{d S=} & \frac{\eta_{y}}{\alpha_{y}}\left(-\frac{S^{0} D}{S^{2}}-\frac{\alpha_{s}}{\eta_{s}} \frac{d x_{s}}{d S}-\frac{\alpha_{I}}{\eta_{I}} \frac{d x_{I}}{d S}\right) \\
= & \frac{\eta_{y}}{\alpha_{y}}\left[-\frac{S^{0} D}{S^{2}}+\frac{\alpha_{s} \alpha_{I}}{\eta_{s} \delta}\right] \\
& -\frac{\alpha_{I} \eta_{y}}{\eta_{I} \alpha_{y}}\left(\frac{\gamma \alpha_{I}\left(-D_{s}+\alpha_{s} S\right)+\alpha_{s}\left(-\alpha_{I} S+D_{I}+\gamma\right)\left(D_{I}-\alpha_{I} S\right)}{\delta\left(D_{I}-\alpha_{I} S\right)^{2}}\right) \\
= & \frac{\eta_{y}}{\alpha_{y}}\left[-\frac{S^{0} D}{S^{2}}-\frac{\alpha_{s} \alpha_{I}}{\delta}\left(\frac{1}{\eta_{I}}-\frac{1}{\eta_{s}}\right)\right] \\
& -\frac{\eta_{y}}{\alpha_{y}}\left[\frac{\alpha_{I} \gamma}{\eta_{I} \delta\left(D_{I}-\alpha_{I} S\right)}\left(\frac{\alpha_{I}\left(-D_{s}+\alpha_{s} S\right)}{\left(D_{I}-\alpha_{I} S\right)}+\alpha_{s}\right)\right] .
\end{aligned}
$$

Recall that the $S$ component of $E_{2}$ must lie in the interval $\left(\frac{D_{s}}{\alpha_{s}}, \frac{D_{I}}{\alpha_{I}}\right)$, and note that if $\eta_{s} \geq \eta_{I}$, the above expression for $\frac{d y}{d S}$ is always negative for $S \in\left(\frac{D_{s}}{\alpha_{s}}, \frac{D_{I}}{\alpha_{I}}\right)$ and $\widehat{S}=\frac{D_{y}}{\alpha_{y}} \in\left(\frac{D_{s}}{\alpha_{s}}, \frac{D_{I}}{\alpha_{I}}\right)$. Therefore, since $y\left(S^{*}\right)=0$, in order for $\widehat{y}>0$, it follows that $\widehat{S}<S^{*}$.

3. If $\eta_{s} \geq \eta_{I}$, and $E_{3}$ exists, then an equilibrium of the form $E_{2}$ must exist by part1. and so by Theorem $3.4, S^{0}>\lambda_{c}$, and by part 2., $S^{*}>\frac{D_{y}}{\alpha_{y}}$. That no other equilibrium can be stable, follows directly from Table 3.1.

If $\eta_{s}<\eta_{I}$, that $E_{1 x}$ or an equilibrium of the form $E_{2}$ can be locally asymptotically stable when $E_{3}$ exists is demonstrted in the example given in Figure 3.7.

B.4. Proofs: Criteria in Table 3.1 - Global Stability for System (2.1). Global stability of $E_{0}$. Assume that $S^{0}<\min \left\{\frac{D_{s}}{\alpha_{s}}, \frac{D_{y}}{\alpha_{y}}\right\}$. The global stability in this case follows immediately from Theorem 3.2 parts 1 ., 3 , and 4 .

REMARK B.3. If $E_{0}$ is locally asymptotically stable, then $E_{0}$ is globally asymptotically stable. Otherwise, for all sufficiently large $t$, for all solutions of (2.1), $0 \leq S(t) \leq S^{0}$.

Global stability of $E_{1 x}$. Consider the Lyapunov function:

$$
\begin{gathered}
V\left(S, x_{s}, x_{I}, y\right)=S-\bar{S}-\bar{S} \ln \left(\frac{S}{\bar{S}}\right)+\frac{1}{\eta_{s}}\left[x_{s}-\bar{x}_{s}-\bar{x}_{s} \ln \left(\frac{x_{s}}{\bar{x}_{s}}\right)\right]+\frac{1}{\eta_{s}} x_{I}+\frac{1}{\eta_{y}} y . \\
\dot{V}=\left(\frac{S-\bar{S}}{S}\right)\left[\left(S^{0}-S\right) D-\frac{\alpha_{s} \bar{x}_{s} S}{\eta_{s}}\right]+\frac{1}{\eta_{s}} x_{I}\left(-D_{I}+\frac{\eta_{s}}{\eta_{I}}\left(\alpha_{I} \bar{S}\right)+\delta \bar{x}_{s}\right) \\
+\frac{1}{\eta_{s}} x_{I}\left[\alpha_{I} S\left(1-\frac{\eta_{s}}{\eta_{I}}\right)-\gamma\left(\frac{\bar{x}_{s}}{x_{s}}\right)\right]-\frac{y}{\eta_{y}}\left(D_{y}-S^{0} \alpha_{y}\right) .
\end{gathered}
$$


Except for the last term involving $y$, the is exactly the same as (B.7) and so, assuming $E_{1 x}$ is locally asymptotically stable, the proof of global stability of $E_{1 x}$ is similar to the proof of global stability of $E_{1^{*}}$, provided that $S^{0}<\frac{D_{y}}{\alpha_{y}}$, so that $y=0$ must hold for $\dot{V}=0$. The same LaSalle Extension Theorem argument applies, with

$$
\Omega=\left\{\left(S, x_{s}, x_{I}, y\right) \in \mathbb{R}_{+}^{4}: S=\bar{S}, x_{I}=0, x_{s} \geq 0, y=0\right\}
$$

and $\mathcal{M}=\left\{\left(\bar{S}, \bar{x}_{s}, 0,0\right)\right\}=\left\{E_{1 x}\right\}$, the largest invariant subset of $\Omega$. Therefore, as for $E_{1^{*}}, E_{1 x}$ is globally asymptotically stable if $S^{0}<\frac{D_{y}}{\alpha_{y}}$ and $D_{I}$ is sufficiently large.

Global stability of $E_{1 y}$. That $E_{1 y}$ is globally asymptotically stable provided that it exists and is locally asymptotically stable follows from Theorem 3.8 part 1., proved in Appendix C.

Global stability of $E_{2}$. Assuming that $E_{2}$ exists and is locally asymptotically stable and that $E_{2^{*}}$ is globally asymptotically stable for (2.2), implies that $E_{2}$ is unique and hence that $R_{0}>1$ by Theorem 3.4. Therefore, by Theorem 3.8 part 6 ., it suffices to show that $y(t) \rightarrow 0$ as $t \rightarrow \infty$. That $y(t) \rightarrow 0$ as $t \rightarrow \infty$ when $\frac{D_{y}}{\alpha_{y}}>\frac{D_{I}}{\alpha_{I}}$, follows from Theorem 3.2 part 2 .

Appendix C. Proofs: Persistence Results.

Proof. (of Theorem 3.8.) 1. Assume that $\frac{D_{y}}{\alpha_{y}}<\frac{D_{s}}{\alpha_{s}}$. Adding the second and third equations of (2.1), we obtain:

$$
\left(x_{s}+x_{I}\right)^{\prime}(t) \leq\left(x_{s}+x_{I}\right)(t)\left(-D_{s}+\alpha_{s} S(t)\right) .
$$

Integrating both sides of the above inequality with respect to $t$ and rearranging,

$$
\int_{0}^{t} S(t) d t \geq \frac{D_{s} t+\ln \left(\left(x_{s}+x_{I}\right)(t)\right)-\ln \left(\left(x_{s}+x_{I}\right)(0)\right)}{\alpha_{s}} .
$$

Integrating both sides of the $y^{\prime}$ equation of (2.1), we again obtain (A.2). Equations (C.1) and (A.2) imply that

$$
\ln (y(t)) \geq \ln (y(0))+\frac{\alpha_{y}}{\alpha_{s}} \ln \left(\frac{\left(x_{s}+x_{I}\right)(t)}{\left(x_{s}+x_{I}\right)(0)}\right)+\alpha_{y} t\left(\frac{D_{s}}{\alpha_{s}}-\frac{D_{y}}{\alpha_{y}}\right) .
$$

By Lemma 3.1, $y(t), x_{s}(t)$, and $x_{I}(t)$ are non-negative and bounded above for all $t \geq 0$, and so $\lim \sup _{t \rightarrow \infty} y(t)<\infty$. Therefore, if $\frac{D_{y}}{\alpha_{y}}<\frac{D_{s}}{\alpha_{s}}$, taking the lim sup on both sides of (C.2), unless $\lim _{t \rightarrow \infty}\left(x_{s}+x_{I}\right)(t)=0$, $\limsup _{t \rightarrow \infty} \ln (y(t))=\infty$, a contradiction.

If in addition, $y(0)>0$ and $S^{0}>\frac{D_{y}}{\alpha_{y}}, E_{1 y}$ is globally asymptotically stable, since either there is a point in the omega limit set of an orbit with $y(0)>0$ of the form $(\widetilde{S}, 0,0, \widetilde{y})$ with $\widetilde{y}>0$, or $\lim _{t \rightarrow \infty} y(t)=0$. In the former case, $E_{1 y}$ must also be in the omega limit set (since on the face where $x_{s} \equiv 0$ and $x_{I} \equiv 0$ the system is a model of growth of a single species in a basic chemostat [38]), but $E_{1 y}$ is locally asymptotically stable with respect to (2.1), and hence it would have to be the only point in the omega limit set. That $\lim _{t \rightarrow \infty} y(t)=0$ is impossible, since then $\lim _{t \rightarrow \infty} S(t)=S^{0}>\frac{D_{y}}{\alpha_{y}}$ and from the $y$ equation of (2.1) it would follow that $y(t)$ must become unbounded, contradicting Lemma 3.1.

2. We prove this result for system (2.1). The proof for subsystem (2.2) is similar. If $x_{I}(t)$ is uniformly strongly persistent, then there exists $\bar{\epsilon}>0$ independent of the 
initial conditions (provided $x_{I}(0)>0$ ), such that for any solution of $(2.1), x_{I_{\infty}}>\bar{\epsilon}$. Applying Lemma A.1 to the $x_{s}$ equation of (2.1), recalling that from Lemma 3.1, $x_{I}^{\infty} \leq \frac{\alpha S^{0}}{A}$, where $\alpha$ and $A$ are defined in the statement of Lemma 3.1,

$$
\begin{aligned}
0 & \geq x_{s_{\infty}}\left(-D_{s}+\alpha_{s} S_{\infty}-\delta x_{I}^{\infty}\right)+\gamma x_{I_{\infty}} \\
& \geq x_{s_{\infty}}\left(-D_{s}-\delta x_{I}^{\infty}\right)+\gamma x_{I_{\infty}} .
\end{aligned}
$$

Rearranging, $x_{s_{\infty}} \geq \frac{\gamma x_{I_{\infty}}}{D_{s}+\delta x_{I}^{\infty}} \geq \frac{\gamma \bar{\epsilon} A}{A D_{s}+\alpha \delta S^{\sigma}}>0$, giving a uniform positive lower bound for $x_{s_{\infty}}$.

3. We prove this result for system (2.1). The proof for subsystem (2.2) is similar. Since $\left(x_{s}+x_{I}\right)(t)$ is strongly persistent, by Theorem 3.2 part $4 ., x_{s}(t)$ must be at least weakly persistent. We continue using proof by contradiction. First suppose that $x_{s}(t)$ is not strongly persistent. Then there exists a solution for which $\lim \sup _{t \rightarrow \infty} x_{s}(t)>0$ and $\liminf \operatorname{in}_{t \rightarrow \infty} x_{s}(t)=0$. Since $\left(x_{s}+x_{I}\right)(t)$ is strongly persistent, for this solution, there exists $\epsilon>0$ such that $\liminf _{t \rightarrow \infty}\left(x_{s}+x_{I}\right)(t)=\epsilon$. Using the Barbălat Lemma [2], there must be a sequence of times $\left\{t_{n}\right\} \rightarrow \infty$ as $t \rightarrow \infty$ such that $x_{s}^{\prime}\left(t_{n}\right)=0$ for all $n, \lim _{n \rightarrow \infty} x_{s}\left(t_{n}\right)=0$, and $\lim _{n \rightarrow \infty} x_{I}\left(t_{n}\right) \geq \epsilon>0$. Consider the $x_{s}$ equation of subsystem (2.2).

$$
0=x_{s}^{\prime}\left(t_{n}\right)=x_{s}\left(t_{n}\right)\left(-D_{s}+\alpha_{s} S\left(t_{n}\right)-\delta x_{I}\left(t_{n}\right)\right)+\gamma x_{I}\left(t_{n}\right) .
$$

Taking the limit as $n \rightarrow \infty$ on both sides, we obtain $0 \geq \gamma \epsilon>0$, a contradiction. Therefore $x_{s}(t)$ is strongly persistent.

Next we proceed using proof by contradiction, to show that if $\left(x_{s}+x_{I}\right)(t)$ is uniformly strongly persistent, then $x_{s}(t)$ is also uniformly strongly persistent. Since $\left(x_{s}+\right.$ $\left.x_{I}\right)(t)$ is uniformly strongly persistent, there exists $\bar{x}>0$ such that $\lim _{\inf _{t \rightarrow \infty}}\left(x_{s}+\right.$ $\left.x_{I}\right)(t) \geq \bar{x}$, independent of initial conditions, provided that $x_{s}(0)>0$ and $x_{I}(0)>0$. Since we just showed that $\left(x_{s}+x_{I}\right)(t)$ strongly persistent implies that $x_{s}(t)$ is strongly persistent, if $x_{s}(t)$ is not uniformly strongly persistent, it follows that there exists a solution $\left(S(t), x_{s}(t), x_{I}(t), y(t)\right)$ with $x_{s}(0)>0$ and (using the Barbălat Theorem [2]), a sequence $\left\{t_{n}\right\}$ with $t_{n} \rightarrow \infty$ as $n \rightarrow \infty$ for which $x_{s}^{\prime}\left(t_{n}\right)=0$ for all $n$, $0<\lim _{n \rightarrow \infty} x_{s}\left(t_{n}\right)=\epsilon_{s}<\min \left\{\bar{x}, \frac{\gamma \bar{x}}{D_{s}+\delta \bar{x}+\gamma}\right\}$, and $\lim _{n \rightarrow \infty} x_{I}\left(t_{n}\right)=\epsilon_{I} \geq \bar{x}-\epsilon_{s}>0$. Taking the limit as $n \rightarrow \infty$ of both sides of the $x_{s}$ equation of system (2.1) evaluated at $t_{n}$, it follows that

$$
0=\epsilon_{s}\left(-D_{s}+\alpha_{s} \lim _{n \rightarrow \infty} S\left(t_{n}\right)\right)+\epsilon_{I}\left(-\delta \epsilon_{s}+\gamma\right) \geq-\epsilon_{s} D_{s}+\epsilon_{I}\left(-\delta \epsilon_{s}+\gamma\right)>0,
$$

a contradiction. To see this note that $\frac{\gamma \epsilon_{I}}{D_{s}+\delta \epsilon_{I}}$ is an increasing function of $\epsilon_{I}$ and since $\epsilon_{I} \geq \bar{x}-\epsilon_{s}$

$$
\frac{\gamma \epsilon_{I}}{D_{s}+\delta \epsilon_{I}} \geq \frac{\gamma\left(\bar{x}-\epsilon_{s}\right)}{D_{s}+\delta\left(\bar{x}-\epsilon_{s}\right)} \geq \frac{\gamma\left(\bar{x}-\epsilon_{s}\right)}{D_{s}+\delta \bar{x}}>\epsilon_{s},
$$

since we assumed that $\epsilon_{s}<\frac{\gamma \bar{x}}{D_{s}+\delta \bar{x}+\gamma}$.

4. Again we prove this result for system (2.1). The proof for subsystem (2.2) is similar. We argue using proof by contradiction. Since $S^{0}<\frac{D_{I}+\gamma}{\alpha_{I}}$, there exists $\epsilon_{1}>0$ such that $S^{0}<\frac{D_{I}+\gamma-\delta \epsilon_{1}}{\alpha_{I}}$. Suppose $x_{s}^{\infty} \leq \epsilon_{1}$. Applying Lemma A.1 to the $x_{I}$ equation of (2.1):

$$
0 \leq x_{I}^{\infty}\left(-D_{I}+\alpha_{I} S^{\infty}+\delta x_{s}^{\infty}-\gamma\right) \leq x_{I}^{\infty}\left[-D_{I}+\alpha_{I} S^{0}+\delta \epsilon_{1}-\gamma\right]<0,
$$


a contradiction, because $x_{I}$ is weakly persistent and the term in the square brackets is negative. Hence $x_{s}>\epsilon_{1}$, independent of the initial conditions, and so $x_{s}$ is uniformly weakly persistent. By Theorem A.2 part 1. with $X=\mathcal{S}$ as defined in Lemma 3.1, $X_{1}=\left\{\left(S, x_{s}, x_{I}, y\right) \in \mathcal{S}: x_{s}>0\right\}$ and $X_{2}=\left\{\left(S, x_{s}, x_{I}, y\right) \in \mathcal{S}: x_{s}=0\right\}$, it follows that $x_{s}$ is uniformly strongly persistent.

5. First consider subsystem (2.2). Since $S^{0}>\frac{D_{s}}{\alpha_{s}}$, select $\bar{\epsilon}>0$ such that $S^{0}-\frac{D_{s}}{\alpha_{s}}-\bar{\epsilon}>0$. We begin by showing that $\left(\frac{\alpha_{s}}{\eta_{s}} x_{s}+\frac{\alpha_{I}}{\eta_{I}} x_{I}\right)^{\infty}>\widetilde{\epsilon}$ where $\widetilde{\epsilon}=$ $\min \left\{\frac{\bar{\epsilon} D}{4 S^{0}}, \frac{\bar{\epsilon} \alpha_{s} \alpha_{I}}{4 \delta \eta_{I}}, \frac{\bar{\epsilon} \alpha_{s}}{4 \delta}\right\}$. Suppose not, i.e., assume that there is some solution of (2.2), such that

$$
\left(\frac{\alpha_{s}}{\eta_{s}} x_{s}+\frac{\alpha_{I}}{\eta_{I}} x_{I}\right)^{\infty} \leq \widetilde{\epsilon}
$$

Applying Lemma A.1 to the $S$ equation of (2.2)

$$
\begin{aligned}
0 & \geq D S^{0}-D S_{\infty}+\liminf _{t \rightarrow \infty}\left[-\left(\frac{\alpha_{s}}{\eta_{s}} x_{s}(t) S_{\infty}+\frac{\alpha_{I}}{\eta_{I}} x_{I}(t) S_{\infty}\right)\right] \\
& \geq D S^{0}-D S_{\infty}-S^{0} \limsup _{t \rightarrow \infty}\left[\frac{\alpha_{s}}{\eta_{s}} x_{s}(t)+\frac{\alpha_{I}}{\eta_{I}} x_{I}(t)\right] \\
& \geq\left(S^{0}-S_{\infty}\right) D-\tilde{\epsilon} S^{0},
\end{aligned}
$$

since $S_{\infty} \leq S^{\infty} \leq S^{0}$ by Lemma 3.1. Therefore, $S_{\infty} \geq S^{0}-\widetilde{\epsilon} \frac{S^{0}}{D}$, and so

$$
S^{0} \geq S^{\infty} \geq S_{\infty} \geq S^{0}-\widetilde{\epsilon} \frac{S^{0}}{D} \geq S^{0}-\frac{\bar{\epsilon}}{4}
$$

Therefore, there exists a $t_{1}>0$ such that $S(t) \in\left[S^{0}-\frac{\bar{\epsilon}}{2}, S^{0}+\frac{\bar{\epsilon}}{2}\right]$ for all $t>t_{1}$. From the $x_{s}$ equation of $(2.2)$ :

$$
\begin{aligned}
x_{s}^{\prime}(t) & \geq x_{s}(t)\left(-D_{s}+\alpha_{s}\left(S^{0}-\frac{\bar{\epsilon}}{2}\right)-\delta x_{I}(t)\right) \\
& \geq x_{s}(t)\left(-D_{s}+\alpha_{s}\left(S^{0}-\frac{\bar{\epsilon}}{2}\right)-\delta\left(\frac{\widetilde{\epsilon} \eta_{I}}{\alpha_{I}}+\widetilde{\epsilon}\right)\right) \\
& \geq \alpha_{s} x_{s}(t)\left(S^{0}-\frac{D_{s}}{\alpha_{s}}-\bar{\epsilon}\right)>0,
\end{aligned}
$$

for all $t>t_{1}$. But this implies that for this solution $x_{s}(t) \rightarrow \infty$ as $t \rightarrow \infty$, contradicting (C.3). Therefore, $\left(\frac{\alpha_{s}}{\eta_{s}} x_{s}+\frac{\alpha_{I}}{\eta_{I}} x_{I}\right)(t)$ or equivalently is $\left(x_{s}+x_{I}\right)(t)$ is uniformly weakly persistent. By Theorem A.2 part 1 . with $X=\mathcal{S}_{(2.2)}, X_{1}=\left\{\left(S, x_{s}, x_{I}\right) \in\right.$ $\mathcal{S}_{(2.2)}: x_{s}>0$ or $\left.x_{I}>0\right\}$ and $X_{2}=\left\{\left(S, x_{s}, x_{I}\right) \in \mathcal{S}_{(2.2)}: x_{s}=0, x_{I}=0\right\}$, it follows that $\left(x_{s}+x_{I}\right)(t)$ is uniformly strongly persistent. That $x_{s}(t)$ is uniformly strongly persistent now follows by part 3 .

Now consider system (2.1) and assume that $\frac{D_{y}}{\alpha_{y}}>\frac{D_{s}}{\alpha_{s}}$. Without loss of generality, assume that $S^{0}>\frac{D_{y}}{\alpha_{y}}$, since otherwise by Theorem 3.2 part 1., $\lim _{t \rightarrow \infty} y(t)=0$ and the proof is similar to the proof for subsystem (2.2), taking $X=\mathcal{S}, X_{1}=$ $\left\{\left(S, x_{s}, x_{I}, y\right) \in \mathcal{S}: y \geq 0\right.$ and, $x_{s}>0$ or $\left.x_{I}>0\right\}$, and $X_{2}=\left\{\left(S, x_{s}, x_{I}, y\right) \in\right.$ $\left.\mathcal{S}: x_{s}=0, x_{I}=0\right\}$. First we show that $x_{s}(t)$ is weakly persistent. If not, then by Theorem 3.2 part 4., there is a solution with $x_{s}(0)>0$, for which $x_{s}(t) \rightarrow 0$ as $t \rightarrow \infty$, and hence by Theorem 3.2 part $4 ., x_{I}(t) \rightarrow 0$ and $y(t) \rightarrow 0$. Since $S^{0}>\frac{D_{y}}{\alpha_{y}}$, there 
exist $\epsilon>0$ such that $S^{0}-\frac{D_{y}}{\alpha_{y}}>\epsilon$. Since $x_{s}(t), x_{I}(t)$ and $y(t) \rightarrow 0$ as $t \rightarrow \infty$, it follows from the $S(t)$ equation of (2.1) that $S(t)>S^{0}-\epsilon$ for all sufficiently large $t$, and hence $y^{\prime}(t)>y(t)\left(-D_{y}+\alpha_{y}\left(S^{0}-\epsilon\right)\right)$. Thus $y(t)$ is eventually growing faster than exponentially, and hence $y(t) \rightarrow \infty$ as $t \rightarrow \infty$, a contradiction. Therefore, $x_{s}(t)$ is weakly persistent.

We apply Theorem A.2 part 2. to prove that $x_{s}(t)$ is uniformly strongly persistent. Define $X=\mathcal{S}, X_{2}=\left\{\left(S, x_{s}, x_{I}, y\right) \in X: x_{s}+x_{I}=0\right\}$, and $X_{1}=X \backslash X_{2}$. Let $x(t)=\left(S(t), x_{s}(t), x_{I}(t), y(t)\right)$ denote a solution of (2.1). Define

$$
Y_{2}=\left\{x(0) \in X_{2}: x(t) \in X_{2}, t>0\right\}=\left\{x(0) \in X: x_{s}(0)+x_{I}(0)=0\right\} .
$$

Since $S^{0}>\frac{D_{s}}{\alpha_{s}}$, the union of omega limit sets of solutions starting in $X_{2}$, denoted by $\Omega_{2}$, is by standard results for growth in the chemostat [38], the set $\left\{E_{0}, E_{1 y}\right\}$, if $\frac{D_{y}}{\alpha_{y}}<S^{0}$ or the set $\left\{E_{0}\right\}$, if $\frac{D_{y}}{\alpha_{y}} \geq S^{0}$. Since $x_{s}(t)$ is weakly persistent, $E_{0}$ and $E_{1 y}$ (when it exists) are weak repellers for $X_{1}$. Therefore, $\left(x_{s}+x_{I}\right)(t)$ is uniformly strongly persistent. That $x_{s}(t)$ is uniformly strongly persistent now follows from part 3.

6. First consider subsystem (2.2). We apply Theorem A.2 part 2. Define $X=\mathcal{S}_{(2.2)}, X_{2}=\left\{\left(S, x_{s}, x_{I}\right) \in X: x_{I}=0\right\}$, and $X_{1}=X \backslash X_{2}$. Let $x(t)=$ $\left(S(t), x_{s}(t), x_{I}(t)\right)$ denote a solution of (2.2). Define

$$
Y_{2}=\left\{x(0) \in X_{2}: x(t) \in X_{2}, t>0\right\}=\left\{x(0) \in X: x_{I}(0)=0\right\} .
$$

Since $S^{0}>\lambda_{c}>\frac{D_{s}}{\alpha_{s}}$ implies that $E_{1 *}$ exists, the union of omega limit sets of solutions starting in $Y_{2}$, denoted by $\Omega_{2}$, is by standard results for single population growth in the chemostat [38], the set $\left\{E_{0^{*}}, E_{1^{*}}\right\}$. We wish to show that if $M_{1}=\left\{E_{0^{*}}\right\}$ and $M_{2}=\left\{E_{1^{*}}\right\}$, then $M=\cup_{i=1}^{2} M_{i}$ is an isolated acyclic covering of $\Omega_{2}$ and that each $M_{i}, i=1,2$ is a weak repeller for $X_{1}$. Since $x_{I}(0)=0$ implies that $x_{I}(t) \equiv 0$, from the basic theory of the chemostat [38], $E_{0 *}$ is globally asymptotically stable with respect to solutions starting in $Y_{2}$ if $x_{s}(0)=0$, and $E_{1 *}$ is globally asymptotically stable with respect to solutions in $Y_{2}$ if $x_{s}(0)>0$. Therefore, $M$ is an isolated acyclic covering of $\Omega_{2}$. If for some solution $x_{I}(0)>0$, then $x_{s}(t)>0$ for all $t>0$. Since $S^{0}>\lambda_{c}>\frac{D_{s}}{\alpha_{S}}$, by part $4 ., x_{s}(t)$ is uniformly strongly persistent. Therefore $M_{1}$ is a weak repeller for $X_{1} . M_{2}$ is also a weak repeller for $X_{1}$, since the third eigenvalue of the Jacobian of $E_{1 *}$ of (2.2) is positive when $S^{0}>\lambda_{c}$, and so $E_{1^{*}}$ is unstable with two dimensional stable manifold given by $W^{+}\left(E_{1^{*}}\right)=\left\{\left(S, x_{s}, x_{I}\right) \in \mathbb{R}^{3}: S \geq 0, x_{I}=0, x_{s}>0\right\}$. Therefore $X_{1}$ does not intersect $W^{+}\left(E_{1 *}\right)$, and so $M_{2}$ is a weak repeller for $X_{1}$. Therefore, by Theorem A.2 part 2., $X_{2}$ is a uniform strong repeller for $X_{1}$, and so $x_{I}(t)$ is also uniformly strongly persistent.

Now consider system $(2.1)$ and assume in addition that $\frac{D_{s}}{\alpha_{s}}<\frac{D_{y}}{\alpha_{y}}$, and $x_{I}(0)>0$ implies that $\lim _{t \rightarrow \infty} y(t)=0$.

We apply Theorem A.2 part 2. Define $X=\mathcal{S}, X_{2}=\left\{\left(S, x_{s}, x_{I}, y\right) \in X: x_{I}=0\right\}$, and $X_{1}=X \backslash X_{2}$. Let $x(t)=\left(S(t), x_{s}(t), x_{I}(t), y(t)\right)$ denote a solution of $(2.1)$. Define

$$
Y_{2}=\left\{x(0) \in X_{2}: x(t) \in X_{2}, t>0\right\}=\left\{x(0) \in X: x_{I}(0)=0\right\} .
$$

Since $S^{0}>\lambda_{c}>\frac{D_{s}}{\alpha_{s}}$ implies that $E_{1 x}$ exists, the union of omega limit sets of solutions starting in $X_{2}$, denoted by $\Omega_{2}$, is by standard results for two population growth in the chemostat [38], the set $\left\{E_{0}, E_{1 x}, E_{1 y}\right\}$, if $\frac{D_{y}}{\alpha_{y}}<S^{0}$ or the set $\left\{E_{0}, E_{1 x}\right\}$, if $\frac{D_{y}}{\alpha_{y}} \geq S^{0}$.

First assume that $\frac{D_{y}}{\alpha_{y}}<S^{0}$. We wish to show that if $M_{1}=\left\{E_{0}\right\}, M_{2}=\left\{E_{1 x}\right\}$, and $M_{3}=\left\{E_{1 y}\right\}$, then $M=\cup_{i=1}^{3} M_{i}$ is an isolated acyclic covering of $\Omega_{2}$ and that 
each $M_{i}, i=1,2,3$ is a weak repeller for $X_{1}$. All three boundary equilibria $E_{0}, E_{1 x}$, and $E_{1 y}$, are unstable in this case with 1,2 , and 3 dimensional stable manifold, respectively. Since $x_{I}(0)=0$ implies that $x_{I}(t) \equiv 0$, from the basic theory of the chemostat [38], the stable manifold of each of these equilibria is given by

$$
\begin{aligned}
W^{+}\left(E_{0}\right) & =\left\{\left(S, x_{s}, x_{I}, y\right) \in \mathbb{R}^{4}: S \geq 0, x_{s}=0, x_{I}=0, y=0\right\} \\
W^{+}\left(E_{1 y}\right) & =\left\{\left(S, x_{s}, x_{I}, y\right) \in \mathbb{R}^{4}: S \geq 0, x_{s}=0, x_{I}=0, y>0\right\} \\
W^{+}\left(E_{1 x}\right) & =\left\{\left(S, x_{s}, x_{I}, y\right) \in \mathbb{R}^{4}: S \geq 0, x_{s}>0, x_{I}=0, y \geq 0\right\} .
\end{aligned}
$$

Therefore, $M$ is an isolated acyclic covering of $\Omega_{2}$. Any solution with $x_{I}(0)>0$ is not in the stable manifold of any of these three equilibria. Using the Butler-McGehee Theorem [28], each $M_{i}$ is a weak repeller for $X_{1}$. Therefore, $X_{2}$ is a uniform strong repeller for $X_{1}$ and hence $x_{I}(t)$ is uniformly strongly persistent. That $x_{s}(t)$ is also uniformly strongly persistent follows from part 2 .

Now assume that $\frac{D_{y}}{\alpha_{y}} \geq S^{0}$. We wish to show that if $M_{1}=\left\{E_{0}\right\}$ and $M_{2}=\left\{E_{1 x}\right\}$ then $M=\cup_{i=1}^{2} M_{i}$ is an isolated acyclic covering of $\Omega_{2}$ and that each $M_{i}, i=1,2$ is a weak repeller for $X_{1}$. In this case, the stable manifold of each of these equilibria is 2 and 3 dimensional respectively, given by

$$
\begin{aligned}
W^{+}\left(E_{0}\right) & =\left\{\left(S, x_{s}, x_{I}, y\right) \in \mathbb{R}^{4}: S \geq 0,: x_{s}=0, x_{I}=0, y \geq 0\right\} \\
W^{+}\left(E_{1 x}\right) & =\left\{\left(S, x_{s}, x_{I}, y\right) \in \mathbb{R}^{4}: S \geq 0, x_{s}>0, x_{I}=0, y \geq 0\right\} .
\end{aligned}
$$

The remainder of the proof is similar to that given for subsystem (2.2).

7. Note that $S^{*}<S^{0}$ and $S^{*}<\frac{D_{I}}{\alpha_{I}}$ always holds whenever $E_{2 *}$ exists. As well, the fourth eigenvalue of the Jacobian of (2.1) evaluated at $E_{2}$ is equal to $-D_{y}+\alpha_{y} S^{*}$, and so is positive since $S^{*}>\frac{D_{y}}{\alpha_{y}}$. Therefore, all four boundary equilibria $E_{0}, E_{1 y}, E_{1 x}$, and $E_{2}$ are unstable in this case, with 1,2, 3, and 3 dimensional table manifolds respectively, given by

$$
\begin{aligned}
W^{+}\left(E_{0}\right) & =\left\{\left(S, x_{s}, x_{I}, y\right) \in \mathbb{R}^{4}: S \geq 0, x_{s}=0, x_{I}=0, y=0\right\} \\
W^{+}\left(E_{1 y}\right) & =\left\{\left(S, x_{s}, x_{I}, y\right) \in \mathbb{R}^{4}: S \geq 0, x_{s}=0, x_{I}=0, y>0\right\} \\
W^{+}\left(E_{1 x}\right) & =\left\{\left(S, x_{s}, x_{I}, y\right) \in \mathbb{R}^{4}: S \geq 0, x_{s}>0, x_{I}=0, y \geq 0\right\} \\
W^{+}\left(E_{2}\right) & =\left\{\left(S, x_{s}, x_{I}, y\right) \in \mathbb{R}^{4}: S \geq 0, x_{s} \geq 0, x_{I}>0, y=0\right\} .
\end{aligned}
$$

Hence, if we define $M_{1}=E_{0}, M_{2}=E_{1 y}, M_{3}=E_{1 x}$, and $M_{4}=E_{2}$, then $M=\cup_{i=1}^{4} M_{i}$ is an isolated covering for $X_{2}=\left\{\left(S, x_{s}, x_{I}, y\right) \in \mathcal{S}: x_{I}=0\right.$ or $\left.y=0\right\}$. Define $X_{1}=\left\{\left(S, x_{s}, x_{I}, y\right) \in \mathcal{S}: x_{I}>0\right.$ and $\left.y>0\right\}$. Then, $X_{1} \cup X_{2}=\mathcal{S}$, and $X_{1}$ is open in $\mathcal{S}$ and forward invariant under the flow of system (2.1). Considering the stable manifold of each equilibrium associated with each $M_{i}$, it is clear using the ButlerMcGehee Theorem [28], that each $M_{i}$ is a weak repeller for $X_{1}$ and the covering $M$ is acyclic. Therefore, by Theorem A.2 part 2., it follows that $X_{2}$ is a uniform strong repeller for $X_{1}$. Hence $x_{I}(t)$ and $y(t)$ are uniformly strongly persistent for system (2.1). Therefore, by part 2., $x_{s}(t)$ is also uniformly strongly persistent for system $(2.1)$.

\section{REFERENCES}

[1] R. Aris And A. E. Humphrey, Dynamics of a chemostat in which two organisms compete for a common substrate, Biotechnol. Bioeng., 19 (1977), pp. 1375-1386. 
[2] I. BARBĂLAT, Systemes d'equations differentielle d'oscillations nonlineaires, Rom. Math. Pures Appl., 4 (1957), pp. 267-270.

[3] E. Beretta, M. Carletti, and F. Solimano, On the effects of environmental fluctuations in a simple model of bactera-bacteriophage infection, Can. Appl. Math. Q., 8 (2000), pp. 321366.

[4] E. Beretta And Y. KuAng, Modeling and analysis of a marine bacteriophage infection with latency period, Nonlinear Anal. Real World Appl., 2 (2001), pp. 35-74.

[5] O. Bergh, K. Y. Borsheim, G. Bratbak, and M. Heldal, High abundance of viruses found in aquatic environments, Nature, 340 (1989), pp. 467-468.

[6] G. Bratbak and M. Heldal, Viruses rule the waves - the smallest and most abundant members of marine ecosystems, Microbiol. Today, 27 (2000), pp. 171-173.

[7] C. P. D. BrussaArd, Role of viruses in controlling phytoplankton blooms, in Ecology of Marine Viruses, vol. 21 of CIEMS Workshop Monographs, CIEMS, 2003.

[8] G. J. Butler and G. S. K. Wolkowicz, A mathematical model of the chemostat with a general class of functions describing nutrient uptake, SIAM J. Appl. Math., 45 (1985), pp. $138-151$.

[9] A. Campbell, Conditions for the existence of bacteriophage, Evolution, 15 (1961), pp. 153-165.

[10] C. Canchaya, C. Proux, G. Fournous, A. Bruttin, and H. Brüssow, Prophage genomics, Microbiol. Mol. Biol. Rev., 67 (2003), pp. 238-276.

[11] C. M. Cavanaugh, Z. P. McKiness, I. L. G. Newton, and F. J. Stewart, Marine chemosynthetic symbioses, in The Prokaryotes. Third Edition. A Handbook on the Biology of Bacteria:Symbiotic Associations, Biotechnology, Applied Microbiology, M. Dworkin, S. Falkow, E. Rosenberg, K. H. Schleifer, and E. Stackebrandt, eds., Springer, New York, 2006, pp. 475-507.

[12] S. P. Daoussis, Predator mediated competition: Predator feeding on two different trophic levels, master's thesis, McMaster University, Hamilton, ON, January 1992.

[13] B. Ermentrout, Simulating, Analyzing, and Animating Dynamical Systems: A Guide to XPPAUT for Researchers and Students, SIAM, Philadelphia, PA, 2002.

[14] S.-B. Hsu, Limiting behavior for competing species, SIAM J. Appl. Math., 34 (1978), pp. 760763.

[15] S. B. Hsu, S. Hubbell, and P. Waltman, A mathematical theory for single-nutrient competition in continuous cultures of micro-organisms, SIAM J. Appl. Math., 32 (1977), pp. 366383.

[16] A. HuRwitz, On the conditions under which an equation has only roots with negative real parts, Math. Ann., 46 (1895), pp. 273-284.

[17] M. Imran and H. L. Smith, A mathematical model of gene transfer in a biofilm, in Mathematics for Ecology and Environmental Sciences, vol. 1 of Biological and Medical Physics, Biomedical Engineering, Springer, Berlin, Heidelberg, 2007, pp. 93-123.

[18] The MathWorks Inc., Matlab, 2010.

[19] A. Larsen, T. Castberg, R. A. Sandaa, C. P. D. Brussaard, J. Egge, M. Heldal, A. Paulino, R. Thyrhaug, E. J. van Hannen, and G. Bratbak, Population dynamics and diversity of phytoplankton, bacteria and viruses in a seawater enclosure, Marine Ecology Progress Series, 221 (2001), pp. 47-57.

[20] B. LI, Global asymptotic behavior of the chemostat: general response functions and differential death rates, SIAM J. Appl. Math., 59 (1999), pp. 411-422.

[21] A. Lwoff, Lysogeny, Bacteriological Rev., 17 (1953), pp. 269-337.

[22] D. Mestivier, K. Pakdaman, P. Y. Boelle, J. C. Nicolas, and P. Lebaron, Viral regulation of bacterial biodiversity, in Ecology of Marine Viruses, vol. 21 of CIEMS Workshop Monographs, 2003.

[23] L. M. Proctor and J. A. Fuhrman, Viral mortality of marine bacteria and cyanobacteria, Nature, 343 (1990), pp. 60-62.

[24] Z. QIU, The analysis and regulation for the dynamics of a temperate bacteriophage model, Math. Biosci., 209 (2007), pp. 417-450.

[25] E.J. Routh, A Treatise on The Stability of Motion, Macmillan, New York, NY, 1877.

[26] A. D. Shabir, J.M. Alfons, Gijs J. K., and M. Gerard, Co-existence of physiologically similar sulfate-reducing bacteria in a full-scale sulfidogenic bioreactor fed with a single organic electron donor, Appl. Microbiol. Biotechnol., 75 (2007), pp. 1463-1472.

[27] H. L. Sмiтh, Models of virulent phage growth with application to phage therapy, SIAM J. Appl. Math., 68 (2008), pp. 1717-1737.

[28] H. L. Smith and P. Waltman, The Theory of the Chemostat, Cambridge University Press, New York, NY, 1995.

[29] — , Perturbation of a globally stable steady state, Proc. Amer. Math. Soc., 127 (1999), 
pp. 447-453.

[30] M. F. Stewart And B. R. Levin, The population biology of bacterial plasmids: a priori conditions for the existence of conjugationally transmitted factors, Genetics, 87 (1977), pp. 209-228.

[31] C. A. Suttle, A. M. Chan, and M. T. Cottrell, Infection of phytoplankton by viruses and reduction of primary productivity, Nature, 347 (1990), pp. 467-469.

[32] M. A. TAPPER AND R. E. Hicks, Temperate viruses and lysogeny in lake superior bacterioplankton, Limnol. Oceanogr., 43 (1998), pp. 95-103.

[33] H. R. Thieme, Persistence under relaxed point-dissipativity, SIAM J. Math. Anal., 24 (1993), pp. 407-435.

[34] P. van den Driessche and M. L. Zeeman, Disease induced oscillations between two competing species, SIAM J. Appl. Math., 3 (2004), pp. 601-619.

[35] J. S Weitz, H. Hartman, and S. A. Levin, Coevolutionary arms races between bacteria and bacteriophage, Proc. Natl. Acad. Sci. USA, 102 (2005), pp. 9535-9540.

[36] C. Westwater, L. M. Kasman, D. A. Schofield, P. A. Werner, J. W. Dolan, M. G. SCHMIDT, AND J. S. NORRIS, Use of genetically engineered phage to deliver antimicrobial agents to bacteria: an alternative therapy for treatment of bacterial infections, Antimicrob. Agents Chemother., (2003), pp. 1301-1307.

[37] G. S. K. Wolkowicz, M. M. Ballyk, and S. P. Daoussis, Interaction in a chemostat: introduction of a competitor can promote greater diversity, Rocky Mountain J. Math., 25 (1995), pp. 515-543.

[38] G. S. K. Wolkowicz And Z. Lu, Global dynamics of a mathematical model of competition in the chemostat: general response functions and differential death rates, SIAM J. Appl. Math., 52 (1992), pp. 222-233. 